\title{
Fixed Points of Self-embeddings of Models of Arithmetic
}

\author{
Saeideh Bahrami \& Ali Enayat
}

July 17, 2018

\begin{abstract}
We investigate the structure of fixed point sets of self-embeddings of models of arithmetic. Our principal results are Theorems A, B, and C below.

In what follows $\mathcal{M}$ is a countable nonstandard model of the fragment $\mathrm{I} \Sigma_{1}$ of PA (Peano Arithmetic); $\mathbb{N}$ is the initial segment of $\mathcal{M}$ consisting of standard numbers of $\mathcal{M} ; \mathrm{I}_{\mathrm{fix}}(j)$ is the longest initial segment of fixed points of $j ; \operatorname{Fix}(j)$ is the fixed point set of $j ; K^{1}(\mathcal{M})$ consists of $\Sigma_{1^{-}}$ definable elements of $\mathcal{M}$; and a self-embedding $j$ of $\mathcal{M}$ is said to be a proper initial self-embedding if $j(\mathcal{M})$ is a proper initial segment of $\mathcal{M}$.
\end{abstract}

Theorem A. The following are equivalent for a proper initial segment I of $\mathcal{M}$ :

(1) $I=\mathrm{I}_{\mathrm{fix}}(j)$ for some self-embedding $j$ of $\mathcal{M}$.

(2) $I$ is closed under exponentiation.

(3) $I=\mathrm{I}_{\mathrm{fix}}(j)$ for some proper initial self-embedding $j$ of $\mathcal{M}$.

Theorem B. The following are equivalent for a proper initial segment I of $\mathcal{M}$ :

(1) $I=\operatorname{Fix}(j)$ for some self-embedding $j$ of $\mathcal{M}$.

(2) $I$ is a strong cut of $\mathcal{M}$ and $I \prec_{\Sigma_{1}} \mathcal{M}$.

(3) $I=\operatorname{Fix}(j)$ for some proper initial self-embedding $j$ of $\mathcal{M}$.

Theorem C. The following are equivalent:

(1) $\operatorname{Fix}(j)=K^{1}(\mathcal{M})$ for some self-embedding $j$ of $\mathcal{M}$.

(2) $\mathbb{N}$ is a strong cut of $\mathcal{M}$.

(3) $\operatorname{Fix}(j)=K^{1}(\mathcal{M})$ for some proper initial self-embedding $j$ of $\mathcal{M}$.

$$
* * * * * * * * * *
$$

2010 Mathematics Subject Classification: Primary 03F30, 03C62, 03H15; Secondary 03C15.

Key Words: Peano Arithmetic, nonstandard model, self-embedding, fixed point, strong cut. 


\section{INTRODUCTION}

In the early 1970s Harvey Friedman [10, Thm. 4.4] proved a remarkable theorem: Every countable nonstandard model $\mathcal{M}$ of PA carries a proper initial self-embedding $j$; i.e., $j$ isomorphically maps $\mathcal{M}$ onto a proper initial segment of $\mathcal{M}$. Friedman's theorem has been generalized and refined in several ways over the past several decades (most recently in [26] and [9]). In the mid-1980s Ressayre [19, and independently Dimitracopoulos \& Paris 3], generalized Friedman's theorem by weakening PA to the fragment $\mathrm{I} \Sigma_{1}$ of PA. In this paper we refine their work by investigating fixed point sets of self-embeddings of countable nonstandard models of I $\Sigma_{1}$.

Our work here was inspired by certain striking results concerning the structure of fixed point sets of automorphisms of countable recursively saturated models of PA summarized in Theorem 1.1 below. In what follows $\mathbb{N}$ is the initial segment of $\mathcal{M}$ consisting of the standard numbers of $\mathcal{M} ; K(\mathcal{M})$ is the set of definable elements of $\mathcal{M} ; \mathrm{I}_{\text {fix }}(j)$ is the longest initial segment of fixed points of $j$; and $\operatorname{Fix}(j)$ is the fixed point set of $j$, in other words:

$$
\begin{gathered}
\mathrm{I}_{\text {fix }}(j):=\{m \in M: \forall x \leq m j(x)=x\}, \text { and } \\
\operatorname{Fix}(j):=\{m \in M: j(m)=m\} .
\end{gathered}
$$

1.1. Theorem. Suppose $\mathcal{M}$ is a countable recursively saturated model of $\mathrm{PA}$, and $I$ is a proper initial segment of $\mathcal{M}$.

(a) (Smoryński [23]) $I=\mathrm{I}_{\mathrm{fix}}(j)$ for some automorphism $j$ of $\mathcal{M}$ iff $I$ is closed under exponentiation 1

(b) (Kaye-Kossak-Kotlarski [15]) $I=\operatorname{Fix}(j)$ for some automorphism $j$ of $\mathcal{M}$ iff ( $I$ is a strong cut of $\mathcal{M}$ and $I \prec \mathcal{M}$ ).

(c) (Kaye-Kossak-Kotlarski [15]) $\operatorname{Fix}(j)=K(\mathcal{M})$ for some automorphism $j$ of $\mathcal{M}$ iff $\mathbb{N}$ is a strong cut of $\mathcal{M} 2$

In this paper we formulate and establish appropriate analogues of each part of Theorem 1.1 for self-embeddings of countable nonstandard models of

\footnotetext{
${ }^{1}$ Smoryński established the right-to-left direction of this result and left the status of the other, much easier direction as an open problem. It is unclear who first established the easier direction, but by now it is considered part of the folklore of the subject. A different proof of (a stronger version of) Smoryński's theorem was established in [6].

${ }^{2}$ This result was generalized in [7] by showing that if $\mathbb{N}$ is strong in $\mathcal{M}$, then the isomorphism types of fixed point sets of automorphisms of $\mathcal{M}$ are precisely the isomorphism types of elementary submodels of $\mathcal{M}$, thus confirming a conjecture of Schmerl.
} 
$\mathrm{I} \Sigma_{1}$, as encapsulated in Theorem 1.2 below. In part (c), $K^{1}(\mathcal{M})$ consists of $\Sigma_{1}$-definable elements of $\mathcal{M}$.

1.2. Theorem. Suppose $\mathcal{M}$ is a countable nonstandard model of $\mathrm{I} \Sigma_{1}$, and $I$ is a proper initial segment of $\mathcal{M}$.

(a) $I=\mathrm{I}_{\mathrm{fix}}(j)$ for some self-embedding $j$ of $\mathcal{M}$ iff $I$ is closed under exponentiation iff $I=\mathrm{I}_{\text {fix }}(j)$ for some proper initial self-embedding $j$ of $\mathcal{M}$.

(b) $I=\operatorname{Fix}(j)$ for some self-embedding $j$ of $\mathcal{M}$ iff ( $I$ is a strong cut of $\mathcal{M}$ and $\left.I \prec_{\Sigma_{1}} \mathcal{M}\right)$ iff $I=\operatorname{Fix}(j)$ for some proper initial self-embedding $j$ of $\mathcal{M}$.

(c) $\operatorname{Fix}(j)=K^{1}(\mathcal{M})$ for some self-embedding $j$ of $\mathcal{M}$ iff $\mathbb{N}$ is a strong cut in $\mathcal{M}$ iff $\operatorname{Fix}(j)=K^{1}(\mathcal{M})$ for some proper initial self-embedding $j$ of $\mathcal{M}$.

The plan of the paper is as follows: Section 2 reviews preliminaries; Section 3 establishes some useful basic results about self-embeddings; and Sections 4, 5, and 6 are respectively devoted to the proofs of parts (a), (b), and (c) of Theorem 1.2. Some further results and open questions are presented in Section 7.

Acknowledgments. Saeideh Bahrami's research was partially supported by the Iranian Ministry of Science, Research \& Technology, and the Department of Philosophy, Linguistics \& Theory of Science of the University of Gothenburg through funds which facilitated her three-month visit to Gothenburg during 2016. Ali Enayat is indebted to Volodya Shavrukov for playing a pivotal role in the inception of this paper since the rudimentary forms of some of the results here were obtained by Enayat in the course of brainstorming email discussions with Volodya during the winter and spring months of 2012. These discussions also led to a number of questions, which were eventually answered in this paper. Both authors are also grateful to Costas Dimitracopoulos, Paul Gorbow, and especially Tin Lok Wong and the anonymous referee for their assistance in weeding out infelicities in earlier drafts of this paper.

\section{PRELIMINARIES}

In this section we review definitions, conventions, and known results that will be utilized in this paper.

- The language of first order arithmetic, $\mathcal{L}_{A}$, is $\{+, \cdot, \mathrm{S}(x),<, 0\}$. $\mathrm{PA}^{-}$ is the $\mathcal{L}_{A}$-theory describing the non-negative parts of discrete ordered 
rings as in [14]. For a language $\mathcal{L} \supseteq \mathcal{L}_{A}, \mathrm{PA}(\mathcal{L})$ is $\mathrm{PA}^{-}$augmented by the induction scheme for all $\mathcal{L}$-formulae. We write $\mathrm{PA}$ for $\operatorname{PA}\left(\mathcal{L}_{A}\right)$; when $\mathcal{L}$ is clear from the context, we shall follow a common practice from the literature and use $\mathrm{PA}^{*}$ to refer to $\operatorname{PA}(\mathcal{L})$.

- $M, M^{*}, M_{0}$, etc. denote (respectively) the universes of discourse of structures $\mathcal{M}, \mathcal{M}^{*}, \mathcal{M}_{0}$, etc. Given an $\mathcal{L}$-structure $\mathcal{M}$ and a class $\Gamma$ of $\mathcal{L}$-formulae, $\operatorname{Th}_{\Gamma}(\mathcal{M})$ is the collection of sentences in $\Gamma$ that hold in $\mathcal{M}$. Also, we write $\operatorname{Th}_{\exists}(\mathcal{M})$ for the collection of existential sentences that hold in $\mathcal{M}$ (an existential formula is of the form $\exists x_{0} \cdots \exists x_{k-1} \varphi$ for quantifier-free $\varphi$ ).

- The meta-theoretic set of natural numbers is here denoted by $\omega$, and we use the notation $\left(a_{i}: i<s\right)$, where $s \in \omega$ or $s=\omega$, to refer to meta-theoretic sequences of finite or infinite length. Given a model $\mathcal{M}$ of $\mathrm{PA}^{-}, \mathbb{N}$ is the initial segment consisting of the standard elements of $\mathcal{M}$. Also, given $s, i$, and $a$ in $\mathcal{M}$, we write $(s)_{i}=a$ to express the fact that $a$ is the $i$-th member of the sequence canonically coded by $s$ in $\mathcal{M}$. In this context, we write $\left\langle a_{i}: i<r\right\rangle$ to refer to the object $s$ in $\mathcal{M}$ such that $s$ is the canonical code in $\mathcal{M}$ of a sequence of length $r$ such that $(s)_{i}=a_{i}$ for each $i<r$. It is well-known [16, Prop. 1.4.1] that we can arrange a canonical coding such that if $s=\left\langle a_{i}: i<r\right\rangle$ and $a_{i}<b$ for all $i<r$, then $s \leq 2^{(r+b+1)^{2}}$.

- For a language $\mathcal{L} \supseteq \mathcal{L}_{A}, \Sigma_{0}(\mathcal{L})=\Pi_{0}(\mathcal{L})=\Delta_{0}(\mathcal{L})=$ the class of $\mathcal{L}$ formulae all of whose quantifiers are of the form $\exists x<t \varphi$ or $\forall x<$ $t \varphi$, where $t$ is an $\mathcal{L}$-term; $\Sigma_{n+1}(\mathcal{L})$ consists of formulae of the form $\exists x_{0} \cdots \exists x_{k-1} \varphi$, where $\varphi \in \Pi_{n}(\mathcal{L})$; and $\Pi_{n+1}(\mathcal{L})$ consists of formulae of the form $\forall x_{0} \cdots \forall x_{k-1} \varphi$, where $\varphi \in \Sigma_{n}(\mathcal{L})$. Here $k$ ranges over $\omega$, with the understanding that $k=0$ corresponds to an empty block of quantifiers. When $\mathcal{L}=\mathcal{L}_{A}$ we write $\Sigma_{n}$ and $\Pi_{n}$ for $\Sigma_{n}(\mathcal{L})$ and $\Pi_{n}(\mathcal{L})$ (respectively).

- For $n \in \omega, \mathrm{I} \Sigma_{n}(\mathcal{L})$ is the fragment of PA with the induction scheme limited to $\Sigma_{n}(\mathcal{L})$-formulae. The $\Sigma_{n}(\mathcal{L})$-Collection Scheme, denoted $\mathrm{B} \Sigma_{n}(\mathcal{L})$, consists of the universal closure of formulae of the following form where $\varphi \in \Sigma_{n}(\mathcal{L})$ and $\varphi$ is allowed to have undisplayed parameters:

$$
[\forall x<v \exists y \varphi(x, y)] \rightarrow \exists z[\forall x<v \exists y<z \varphi(x, y)] .
$$


- Given a theory $T$, and a class $\Gamma$ of formulae, $\Gamma^{T}$ is the class of formulae that are $T$-provably equivalent to some formula in $\Gamma$. It is well-known [14, Ch. 7] that $\Sigma_{n}^{T}$ and $\Pi_{n}^{T}$ are both closed under bounded quantification, disjunction, and conjunction for $T=\mathrm{I} \Delta_{0}+\mathrm{B} \Sigma_{n}$.

- For models $\mathcal{M}$ and $\mathcal{N}$ of $\mathcal{L}_{A}$, we say that $\mathcal{N}$ end extends $\mathcal{M}$ (equivalently: $\mathcal{M}$ is an initial submodel of $\mathcal{N}$ ), if $\mathcal{M}$ is a submodel of $\mathcal{N}$ and $a<b$ for every $a \in M$, and $b \in N \backslash M$. For a class $\Gamma$ of $\mathcal{L}$-formulae we write $\mathcal{M} \prec_{\Gamma} \mathcal{N}$ if $\mathcal{N}$ is a $\Gamma$-elementary extension of $\mathcal{M}$, i.e., $\Gamma$-formulae with parameters in $\mathcal{M}$ are absolute in the passage between $\mathcal{M}$ and $\mathcal{N}$. An embedding $\mathcal{M}$ into $\mathcal{N}$ is an isomorphism $j$ between $\mathcal{M}$ and a submodel of $\mathcal{N}$; such an embedding $j$ is said to be an initial embedding if the range of $j$ is an initial segment of $\mathcal{N}$. An initial self-embedding of $\mathcal{M}$ is an initial embedding of $\mathcal{M}$ into itself. A self-embedding $j$ is proper if $j$ is not surjective (equivalently, if $j$ is not an automorphism), otherwise $j$ is said to be improper. Also, we say that a self-embedding $j$ is trivial if $j$ is the identity map on $\mathcal{M}$; otherwise $j$ is nontrivial. Under these definitions, every automorphism of $\mathcal{M}$ is an improper initial self-embedding; and every proper self-embedding is nontrivial.

- $\mathrm{ACA}_{0}$ is the well-known subsystem of second order arithmetic with the comprehension scheme limited to formulae with no second order quantifiers, as in [20]. Models of $\mathrm{ACA}_{0}$ are of the two-sorted form $(\mathcal{M}, \mathcal{A})$, where $\mathcal{A}$ is a family of subsets of $M,(\mathcal{M}, S)_{S \in \mathcal{A}} \models \mathrm{PA}^{*}$, and $\mathcal{A}$ is closed under arithmetical definability. $\mathrm{WKL}_{0}$ is a subsystem of $\mathrm{ACA}_{0}$ whose models are of the form $(\mathcal{M}, \mathcal{A})$, where $(\mathcal{M}, \mathcal{A})$ satisfies (1) Induction for $\Sigma_{1}^{0}$ formulae (where $\Sigma_{1}^{0}$ is the family of $\Sigma_{1}(\mathcal{L}(\mathcal{A})$ ) formulae with no second order quantifier); (2) Comprehension for $\Delta_{1}^{0}$ formulae; and (3) Weak König's Lemma (which asserts that every infinite subtree of the full binary tree has an infinite branch).

The following result is due to Paris and Pudlák; it refines Bennett's celebrated result stating that the graph of the exponential function $y=2^{x}$ is definable by a $\Delta_{0}$-predicate in the standard model of arithmetic. See [13, Sec. V3(c)] for further detail.

2.1. Theorem. (Paris, Pudlák) There is a $\Delta_{0}$-formula $\operatorname{Exp}(x, y)$ such that $\mathrm{I} \Delta_{0}$ proves the following three statements:

(a) $\forall x \exists \leq 1 y \operatorname{Exp}(x, y)$.

(b) $\forall x(\exists y \operatorname{Exp}(x, y) \rightarrow \forall z<x \exists y \operatorname{Exp}(z, y))$.

(c) $\forall x \forall y(\operatorname{Exp}(x, y) \rightarrow \operatorname{Exp}(x+1,2 y))$. 
- I $\Delta_{0}+\operatorname{Exp}$ is the extension of $\mathrm{I} \Delta_{0}$ obtained by adding the axiom Exp, where $\operatorname{Exp}:=\forall x \exists y \operatorname{Exp}(x, y)$. The theory $\mathrm{I} \Delta_{0}+\operatorname{Exp}$ might not appear to be particularly strong since it cannot even prove the totality of the superexponential function, but experience has shown that it is a remarkably robust theory that is able to prove an extensive array of theorems of number theory and finite combinatorics.

- A cut $I$ of a model $\mathcal{M}$ of $\mathrm{PA}^{-}$is an initial segment of $\mathcal{M}$ with no last element. We write $m<I$, where $m \in M$, to indicate that some member of $I$ exceeds $m$. Similarly, we write $I<m$ to indicate that every member of $I$ is below $m$. When a cut $I$ is closed under multiplication (and therefore under addition as well), we shall use $I$ also to refer to the submodel of the ambient model whose universe is $I$.

The following result is folklore; the verification that $\mathrm{I} \Delta_{0}$ holds in $I$ is done by a routine induction on the length of $\Delta_{0}$-formulae; see [14, Prop. 10.5 $(n=1)]$ for a proof that $\mathrm{B} \Sigma_{1}$ holds in $I$.

2.2. Theorem. If $I$ is a proper cut of a model of $\mathrm{I} \Delta_{0}$ and $I$ is closed under multiplication, then $I \models \mathrm{I} \Delta_{0}+\mathrm{B} \Sigma_{1}$.

- We will use $E$ to denote Ackermann's membership relation defined by: $x E y$ iff the $x$-th bit of the binary expansion of $y$ is a 1 . It is well-known that within $\mathrm{I} \Delta_{0}+\operatorname{Exp}$ the formula $x E y$ is equivalent to a $\Delta_{0}$-formula. A subset $X$ of $M$ is coded in $\mathcal{M}$ iff for some $m \in M$,

$$
X=\left(m_{E}\right)^{\mathcal{M}}:=\{x \in M: \mathcal{M} \models x E m\} .
$$

- Given $m \in M, \underline{m}^{\mathcal{M}}:=\left\{x \in M: x<^{\mathcal{M}} m\right\}$. Note that $\underline{m}$ is coded in $\mathcal{M} \models \mathrm{I} \Delta_{0}$ provided $2^{m}$ exists in $\mathcal{M}$. When $\mathcal{M}$ is clear from the context, we simply write $\underline{m}$ for $\underline{m}^{\mathcal{M}}$.

- $X$ is piece-wise coded in $\mathcal{M}$ if $\underline{m} \cap X$ is coded in $\mathcal{M}$ for each $m$ in $\mathcal{M}$.

- For a cut $I$ of $\mathcal{M}, \mathrm{SSy}_{I}(\mathcal{M})$ is the family consisting of sets of the form $S \cap I$, where $S$ is a subset of $M$ that is coded in $\mathcal{M}$, i.e.,

$$
\operatorname{SSy}_{I}(\mathcal{M})=\left\{\left(c_{E}\right)^{\mathcal{M}} \cap I: c \in M\right\} .
$$

When $I=\mathbb{N}$, we shall write the commonly used notation $\operatorname{SSy}(\mathcal{M})$ instead of $\operatorname{SSy}_{\mathbb{N}}(\mathcal{M})$. It is well-known [2, Cor. 3.1] that $(\mathbb{N}, \operatorname{SSy}(\mathcal{M})) \models$ $\mathrm{WKL}_{0}$ for a nonstandard $\mathcal{M} \models \mathrm{I} \Delta_{0}$; in $\operatorname{particular} \operatorname{SSy}(\mathcal{M})$ is a Boolean algebra and closed under Turing reducibility. 
- $\Delta_{0}\left(\Sigma_{n}\right)$ is the class of $\mathcal{L}_{A}$-formulae obtained by closing the class of $\Sigma_{n}$-formulae under Boolean connectives and bounded quantifiers.

- For a formula $\varphi\left(x_{1}, \cdots, x_{k}\right)$ whose free variables are ordered as shown, we write $\varphi^{\mathcal{M}}$ for $\left\{\left(m_{1}, \cdots, m_{k}\right) \in M^{k}: \mathcal{M} \models \varphi\left(m_{1}, \cdots, m_{k}\right)\right\}$.

- Given a class $\Gamma$ of formulae, the $\Gamma$-Strong Collection Scheme, here denoted $\mathrm{B}^{+} \Gamma$, consists of the universal closure of formulae of the following form, where $\varphi(x, y) \in \Gamma$ and $\varphi$ is allowed to have undisplayed parameters:

$$
\exists z \forall x<v[\exists y \varphi(x, y) \rightarrow \exists y<z \varphi(x, y)] .
$$

- Sat $_{\Sigma_{n}}$ is the $\mathcal{L}_{\mathrm{A}}$-formula defining the satisfaction predicate for $\Sigma_{n^{-}}$ formulae for an ambient model satisfying $\mathrm{I} \Delta_{0}+$ Exp. It is well-known that $\operatorname{Sat}_{\Sigma_{n}} \in \Sigma_{n}^{\mathrm{I} \Sigma_{1}}$ for each positive $n \in \omega$, and $\operatorname{Sat}_{\Sigma_{0}} \in \Sigma_{1}^{\mathrm{I} \Sigma_{1}}$ [13, Thm. 1.75].

The following theorem collects together a number of important properties of models of $\mathcal{M}=\mathrm{I} \Sigma_{n}$; see [13, Ch. I] for an exposition.

2.3. Theorem. If $n \in \omega, \mathcal{M} \models \mathrm{I} \Sigma_{n}$, and $\varphi$ is a unary $\Delta_{0}\left(\Sigma_{n}\right)$-formula $\varphi(x, a)$, where $a$ is a parameter from $\mathcal{M}$, then:

(a) If $n>0$, then $\mathcal{M} \models \mathrm{B}^{+}\left(\Sigma_{n}\right)$.

(b) $\varphi^{\mathcal{M}}$ is piece-wise coded in $\mathcal{M}$ if $n>0$, or if $n=0$ and $\mathcal{M} \models$ Exp.

(c) $\left[\Delta_{0}\left(\Sigma_{n}\right)\right.$-Min] If $\varphi^{\mathcal{M}}$ is nonempty, then $\varphi^{\mathcal{M}}$ has a minimum element.

(d) $\left[\Delta_{0}\left(\Sigma_{n}\right)-\mathrm{Max}\right]$ If $\varphi^{\mathcal{M}}$ is nonempty and bounded in $\mathcal{M}$, then $\varphi^{\mathcal{M}}$ has a maximum element.

(e) $\left[\Delta_{0}\left(\Sigma_{n}\right)\right.$-Overspill] If $\varphi^{\mathcal{M}}$ includes a proper cut I of $\mathcal{M}$, then $\underline{m} \subseteq \varphi^{\mathcal{M}}$ for some $m>I$.

(f) $\left[\Delta_{0}\left(\Sigma_{n}\right)-\mathrm{PHP}\right]$ If $n>0$ and $\varphi^{\mathcal{M}}$ is the graph of a function $f$ from $\underline{m+1}$ into $\underline{m}$, then $f$ is not one-to-one.

2.3.1. Remark. Suppose $\mathcal{M}$ is a nonstandard model of $\mathrm{I} \Sigma_{n}$ for $n>0$, and $p(x)$ is a collection of formulae $\varphi(x, a)$ (where $a$ is a parameter in $\mathcal{M}$ ) such that (1) $p(x)$ is a $\Sigma_{n}$-type (i.e., every $\varphi \in p(x)$ is a $\Sigma_{n}$-formula); or (2) $p(x)$ is a short $\Pi_{n}$-type (i.e., $p(x)$ includes the formula $x<(a)_{i}$ for some $i \in \omega$, and every $\varphi \in p(x)$ is a $\Pi_{n}$-formula). Then using part (e) of Theorem 2.3 (with $I=\mathbb{N}$ ), and the fact that Sat $_{\Sigma_{n}}$ has a $\Sigma_{n}$-description in $\mathcal{M}$ it is routine to verify that if $p(x)$ is coded in $\mathcal{M}$ (i.e., $\{\ulcorner\varphi(x, y)\urcorner: \varphi \in p(x)\} \in \operatorname{SSy}(\mathcal{M})$ ) and $p(x)$ is finitely realizable in $\mathcal{M}$, then $p(x)$ is realized in $\mathcal{M}$. 
- Given a class $\Gamma$ of formulae and $\mathcal{M} \models \mathrm{PA}^{-}, m \in M$ is said to be $\Gamma$-definable in $\mathcal{M}$ if $\{m\}=\gamma^{\mathcal{M}}$ for some unary $\gamma(x) \in \Gamma$; and $m$ is $\Gamma$-minimal in $\mathcal{M}$ if there is unary $\gamma(x) \in \Gamma$ such that $m$ is the first element of $\gamma^{\mathcal{M}}$. Note that $m$ is $\Delta_{0}$-definable iff $m$ is $\Delta_{0}$-minimal. In general, if $m$ is $\Gamma$-definable then $m$ is $\Gamma$-minimal (but not conversely).

- Given $\mathcal{M} \models \mathrm{PA}^{-}, K^{n}(\mathcal{M})$ is the submodel of $M$ whose universe consists of all $\Sigma_{n}$-definable elements of $\mathcal{M}$. The following result was originally proved by Paris \& Kirby [18, Prop. 8]; see [13, Ch. IV] for an expository account.

2.4. Theorem. (Paris \& Kirby) Suppose $n \in \omega$ and $\mathcal{M} \models \mathrm{I} \Sigma_{n+1}$.

(a) $K^{n+1}(\mathcal{M}) \prec_{\Sigma_{n+1}} \mathcal{M}$.

(b) $K^{n+1}(\mathcal{M}) \models \mathrm{I} \Sigma_{n}+\neg \mathrm{B} \Sigma_{n+1}$, if $K^{n+1}(\mathcal{M})$ is nonstandard.

- Given a cut $I$ of $\mathcal{M}, I$ is said to be a strong cut of $\mathcal{M}$ if, for each function $f$ whose graph is coded in $\mathcal{M}$ and whose domain includes $I$, there is some $s$ in $M$ such that for all $m \in I, f(m) \notin I$ iff $s<f(m)$. Paris \& Kirby proved that strong cuts of models of PA are themselves models of PA [18, Prop. 8]. Indeed, their proof shows the following more general result (see [16, Sec. 7.3] or [5, Lem. A.4]).

2.5. Theorem. (Paris \& Kirby) The following are equivalent for a proper cut $I$ of $\mathcal{M} \models \mathrm{I} \Delta_{0}$ :

(1) $I$ is a strong cut of $\mathcal{M}$.

(2) $\left(I, \operatorname{SSy}_{I}(\mathcal{M})\right) \models \mathrm{ACA}_{0}$.

- Given a linearly ordered structure $\mathcal{K}$, let $\operatorname{Aut}(\mathcal{K})$ be the automorphism group of $\mathcal{K} ; \operatorname{SE}(\mathcal{K})$ be the semi-group of self-embeddings of $\mathcal{K} ; \operatorname{ISE}(\mathcal{K})$ be the semi-group of initial self-embeddings of $\mathcal{K}$, and $\operatorname{PISE}(\mathcal{K})$ be the semi-group of all proper initial self-embeddings of $\mathcal{K}$ (all under composition). Also, a self-embedding $j$ of $\mathcal{K}$ is contractive iff $j(a) \leq a$ for all $a \in K$.

Theorem 2.6 below summarizes some remarkable results of Gaifman [11, Thm. 4.9-4.11]; his results were couched in terms of arbitrary models of $\operatorname{PA}(\mathcal{L})$ for countable $\mathcal{L}$ and are proved using the technology of 'minimal types' 3 A streamlined proof of part (a) and the right-to-left direction of

\footnotetext{
${ }^{3}$ Note that if $(\mathcal{M}, \mathcal{A}) \models \mathrm{ACA}_{0}$, then the expansion $(\mathcal{M}, A)_{A \in \mathcal{A}}$ of $\mathcal{M}$ is a model of $\operatorname{PA}(\mathcal{L})$, where $\mathcal{L}$ is the extension of $\mathcal{L}_{A}$ by predicate symbols for each $A \in \mathcal{A}$. Moreover, the collection of subsets of $M$ that are parametrically definable in $(\mathcal{M}, A)_{A \in \mathcal{A}}$ coincides with $\mathcal{A}$.
} 
part (e) appears in [5, Thm. B]. Part (h) of Theorem 2.6 seems to be absent in Gaifman's paper; but a proof can be found in [7, Thm. 3.3.8(c)]; the proof there is written for $j \in \operatorname{Aut}(\mathbb{L})$, but the reasoning carries over for $j \in \operatorname{SE}(\mathbb{L})$.

2.6. Theorem. (Gaifman) Suppose $(\mathcal{M}, \mathcal{A})$ is a countable model of $\mathrm{ACA}_{0}$. Given any linear order $\mathbb{L}$, there is $\mathcal{N}_{\mathbb{L}} \succ_{\text {end }} \mathcal{M}$ and an isomorphic copy $\mathbb{L}^{\prime}=\left\{c_{l}: l \in \mathbb{L}\right\}$ of $\mathbb{L}$ in $N_{\mathbb{L}} \backslash M$, along with a composition preserving embedding $j \mapsto \widehat{j}$ of $\operatorname{SE}(\mathbb{L})$ into $\operatorname{SE}\left(\mathcal{N}_{\mathbb{L}}\right)$ such that:

(a) $\operatorname{SSy}_{M}\left(\mathcal{N}_{\mathbb{L}}\right)=\mathcal{A}$ and $M \subseteq \operatorname{Fix}(\widehat{j})$ for each $j \in \operatorname{SE}(\mathbb{L})$; moreover $M=$ $\operatorname{Fix}(\widehat{j})$ iff $j$ is fixed point free.

(b) $\widehat{j}$ is an elementary self-embedding of $\mathcal{N}_{\mathbb{L}}$ for each $j \in \mathrm{SE}(\mathbb{L})$.

(c) $\mathbb{L}^{\prime}$ is downward cofinal in $N_{\mathbb{L}} \backslash M$ if $\mathbb{L}$ has no first element.

(d) For any $l_{0} \in \mathbb{L}, l_{0}$ is a strict upper bound for $j(\mathbb{L})$ iff $c_{l_{0}}$ is a strict upper bound for $\widehat{j}\left(N_{\mathbb{L}}\right)$.

(e) $\widehat{j} \in \operatorname{Aut}\left(\mathcal{N}_{\mathbb{L}}\right)$ iff $j \in \operatorname{Aut}(\mathbb{L})$.

(f) $\widehat{j} \in \operatorname{ISE}\left(\mathcal{N}_{\mathbb{L}}\right)$ iff $j \in \operatorname{ISE}(\mathbb{L})$.

(g) $\hat{j} \in \operatorname{PISE}\left(\mathcal{N}_{\mathbb{L}}\right)$ iff $j \in \operatorname{PISE}(\mathbb{L})$.

(h) $\widehat{j}$ is contractive iff $j$ is contractive.

The following is Smoryński's refinement of Friedman's embedding theorem. The proof is outlined in [21, Thm. 3.9], and given in detail in [22, Thm. 2.4] (Smoryński proved his result for countable nonstandard models of PA; but the proof readily goes through for countable nonstandard models of $\left.\mathrm{I} \Sigma_{1}\right)$.

2.7. Theorem. (Smoryński) Suppose $\mathcal{M}$ and $\mathcal{N}$ are countable nonstandard models of $\mathrm{I} \Sigma_{1}$. The following are equivalent:

(1) There is an embedding of $\mathcal{M}$ into $\mathcal{N}$.

(2) $\operatorname{SSy}(\mathcal{M}) \subseteq \operatorname{SSy}(\mathcal{N})$ and $\operatorname{Th}_{\Sigma_{1}}(\mathcal{M}) \subseteq \operatorname{Th}_{\Sigma_{1}}(\mathcal{N})$.

(3) There is an embedding $j$ of $\mathcal{M}$ into $\mathcal{N}$ such that $j(\mathcal{M})$ is a 'mixed' submodel of $\mathcal{N}$, i.e., $j(\mathcal{M})$ is neither cofinal in $\mathcal{M}$ nor an initial segment of $\mathcal{N}$.

2.7.1. Remark. As noted by Smoryński [24, p. 21] the condition $\operatorname{Th}_{\Sigma_{1}}(\mathcal{M}) \subseteq$ $\operatorname{Th}_{\Sigma_{1}}(\mathcal{N})$ in $(2)$ above can be weakened to $\operatorname{Th}_{\exists}(\mathcal{M}) \subseteq \operatorname{Th}_{\exists}(\mathcal{N})$, thanks to the MRDP Theorem. The MRDP Theorem (due to Matijasevič, Robinson, Davis, and Putnam) states that every recursively enumerable set is Diophantine. As shown by Dimitracopoulos and Gaifman [4] the MRDP Theorem is provable in $\mathrm{I} \Delta_{0}+\operatorname{Exp}$. 
The next result is due to Wilkie (according to [22, where it first appeared in print). Wilkie's result was formulated for countable nonstandard models of PA, but an inspection of the proof presented in [14, Thm. 12.6] makes it clear that the result holds for countable nonstandard models of $\mathrm{I} \Sigma_{2}$.

2.8. Theorem. (Wilkie) Suppose $\mathcal{M}$ and $\mathcal{N}$ are countable nonstandard models of $\mathrm{I} \Sigma_{2}$. The following are equivalent:

(1) For each $a \in N$ there is a proper initial embedding $j$ of $\mathcal{M}$ into $\mathcal{N}$ such that $a \in j(M)$.

(2) $\operatorname{SSy}(\mathcal{M})=\operatorname{SSy}(\mathcal{N})$ and $\operatorname{Th}_{\Pi_{2}}(\mathcal{M}) \subseteq \operatorname{Th}_{\Pi_{2}}(\mathcal{N})$.

The following result of Ressayre [19] shows that all countable nonstandard models of $I \Sigma_{1}$ carry proper initial self-embeddings that pointwise fix any prescribed topped initial segment; and $I \Sigma_{1}$ is the weakest extension of $\mathrm{I} \Delta_{0}$ with this property. The $(1) \Rightarrow(2)$ direction of Ressayre's theorem is refined in Corollary 3.3.1 and Theorem 4.1; see Remarks 3.3.2 and 4.1.2 for more detail.

2.9. Theorem. (Ressayre) The following are equivalent for a countable nonstandard $\mathcal{M} \models \mathrm{I} \Delta_{0}$ :

(1) $\mathcal{M} \models \mathrm{I} \Sigma_{1}$.

(2) For each $a \in M$, there is a proper initial self-embedding $j$ of $\mathcal{M}$ such that $j(m)=m$ for each $m \leq a$.

\section{BASIC RESULTS}

In this section we establish a number of basic results about self-embeddings. These results will also be useful in subsequent sections.

3.1. Theorem. Suppose $j$ is a self-embedding of $\mathcal{M} \models \mathrm{I} \Delta_{0}+$ Exp. Then $K^{1}(\mathcal{M}) \preceq_{\Sigma_{1}} \operatorname{Fix}(j) \preceq \Sigma_{1} \mathcal{M}$.

Before presenting the proof of Theorem 3.1, we will establish two useful lemmas.

3.1.1. Lemma. If $\mathcal{M}$ and $\mathcal{N}$ are both models of $\mathrm{I} \Delta_{0}+$ Exp, and $j$ is an embedding of $\mathcal{M}$ into $\mathcal{N}$, then $j(\mathcal{M}) \preceq_{\Delta_{0}} \mathcal{N}$.

Proof. If $j$ is an initial embedding, then this follows from the basic fact that every submodel of $\mathcal{N}$ whose universe is a cut of $\mathcal{N}$ that is closed under multiplication (and therefore addition) is a $\Delta_{0}$-elementary submodel of $\mathcal{N}$. 
For the general case, this follows from the provability of the MRDP Theorem in models of I $\Delta_{0}+\operatorname{Exp}$, since if $\mathcal{N}_{0}$ is a submodel of $\mathcal{N}$, where both $\mathcal{N}_{0}$ and $\mathcal{N}$ are models of $\mathrm{I} \Delta_{0}+\mathrm{MRDP}$, then $\mathcal{N}_{0} \preceq_{\Delta_{0}} \mathcal{N}$.

3.1.2. Lemma. Suppose $\mathcal{M} \models \mathrm{I} \Delta_{0}$.

(a) If $D$ is a nonempty $\Sigma_{1}$-definable subset of $\mathcal{M}$, then there is some $d \in D$ such that $d$ is $\Delta_{0}$-minimal in $(\mathcal{M}, m)$ for some $\Delta_{0}$-minimal element $m$ of $\mathcal{M}$ with $d<m$.

(b) If $d \in K^{1}(\mathcal{M})$, then $d$ is $\Delta_{0}$-minimal in $(\mathcal{M}, m)$ for some $\Delta_{0}$-minimal element $m$ of $\mathcal{M}$ with $d<m$.

(c) If in addition $\mathcal{M} \models$ Exp, and $j$ is a self-embedding of $\mathcal{M}$ such that $j(m)=m$, and $d$ is $\Delta_{0}$-minimal in $(\mathcal{M}, m)$, then $j(d)=d$.

Proof. (a) Easy; suppose $D$ is definable by the formula $\exists z \delta(x, z)$, where $\delta$ is $\Delta_{0}$. Let $m$ be the first element in $\mathcal{M}$ such that $\delta(x, z)$ holds for some $x$ and $z$ below $m$, and then let $d$ be the first element below $m$ such that $\delta(d, z)$ holds for some $z<m$.

(b) This follows immediately from part (a) by setting $D=\{d\}$.

(c) Suppose $\delta(x, y)$ is a $\Delta_{0}$-formula such that:

(1) $(\mathcal{M}, m) \models d=\mu x \delta(x, m)$,

where $\mu$ is the least search operator. (1) coupled with the assumption that $j$ is an isomorphism between $\mathcal{M}$ and $j(\mathcal{M})$ implies:

(2) $(j(\mathcal{M}), j(m)) \models j(d)=\mu x \delta(x, j(m))$.

By putting (2) together with $j(\mathcal{M}) \preceq_{\Delta_{0}} \mathcal{M}$ (by Lemma 3.1.1) and the assumption $j(m)=m$ we have:

(3) $(\mathcal{M}, m) \models j(d)=\mu x \delta(x, m)$.

By putting (1) together with (3) we can now conclude that $j(d)=d$.

Proof of Theorem 3.1. Let us first establish $\operatorname{Fix}(j) \preceq_{\Sigma_{1}} \mathcal{M}$. By Tarski's test, it suffices to show that for every $\Delta_{0}$-formula $\delta(x, y)$, if $\mathcal{M} \models \exists x \delta(x, m)$ for some $m \in \operatorname{Fix}(j)$, then $\mathcal{M} \models \delta(d, m)$ for some $d \in \operatorname{Fix}(j)$. Let $D$ be defined in $\mathcal{M}$ as consisting of elements $x$ such that $\delta(x, m)$, and let $d$ be the least member of $D$. Then $d$ is $\Delta_{0}$-minimal in $(\mathcal{M}, m)$, and therefore $j(d)=d$ by part (c) of Lemma 3.1.2.

To see that $K^{1}(\mathcal{M}) \subseteq \operatorname{Fix}(j)$, suppose $d \in K^{1}(\mathcal{M})$. Then by part (b) of Lemma 3.1.2 there is some $\Delta_{0}$-minimal element $m$ of $\mathcal{M}$ such that $d$ is $\Delta_{0}$-minimal in $(\mathcal{M}, m)$. Therefore by two applications of part (c) of Lemma 3.1 .2 we can obtain $j(m)=m$ and $j(d)=d$. Recall that $K^{1}(\mathcal{M}) \preceq_{\Sigma_{1}} \mathcal{M}$ (by the $n=0$ case of Theorem 2.4), and we have already verified that 
$K^{1}(\mathcal{M}) \subseteq \operatorname{Fix}(j)$ and $\operatorname{Fix}(j) \preceq_{\Sigma_{1}} \mathcal{M}$. On the other hand it can be easily seen that in general if $\mathcal{N}_{0}$ and $\mathcal{N}_{1}$ are $\Sigma_{1}$-elementary submodels of an $\mathcal{L}_{A^{-}}$ structure $\mathcal{N}$ with $N_{0} \subseteq N_{1}$, then $\mathcal{N}_{0} \preceq \Sigma_{1} \mathcal{N}_{1}$. This completes the proof of $K^{1}(\mathcal{M}) \preceq_{\Sigma_{1}} \operatorname{Fix}(j)$.

3.1.3. Remark. It is easy to see, using part (b) of Lemma 3.1.2, that $K^{1}(\mathcal{M})=\Delta_{1}^{\mathcal{M}}$; i.e., the elements of $K^{1}(\mathcal{M})$ are precisely those elements of $\mathcal{M}$ that are both $\Sigma_{1}$-definable and $\Pi_{1}$-definable in $\mathcal{M}$. This observation dates back to Mijajlović [17].

The following result generalizes the $(a) \Rightarrow(b)$ direction of [6, Thm. A], which corresponds to Theorem 3.2 when $j$ is a nontrivial automorphism of $\mathcal{M}$.

3.2. Theorem. If $\mathcal{M} \models \mathrm{I} \Delta_{0}$ and $j$ is a nontrivial self-embedding of $\mathcal{M}$ such that $j(\mathcal{M}) \preceq_{\Delta_{0}} \mathcal{M}$, then $\mathrm{I}_{\text {fix }}(j) \models \mathrm{I} \Delta_{0}+\mathrm{B} \Sigma_{1}+$ Exp.

Proof. We first verify that $\mathrm{I}_{\mathrm{fix}}(j)$ is closed under the operations of the ambient structure $\mathcal{M}$. Suppose $x$ and $y$ are elements of $\mathrm{I}_{\text {fix }}(j)$ with $x \leq y$ and, without loss of generality, assume that $x$ and $y$ are both nonstandard elements. Since $x+y<x y \leq y^{2}$, it suffices to show that $y^{2} \in \mathrm{I}_{\mathrm{fix}}(j)$. Observe that $I \Delta_{0}$ can prove that any number $z<y^{2}$ can be written as $z=q y+r$, where both $q$ and $r$ are less than $y$ (since the division algorithm can be implemented in $\left.\mathrm{I} \Delta_{0}\right)$. Therefore,

$$
j(z)=j(q y+r)=j(q) j(y)+j(r)=q y+r=z .
$$

This shows that $\mathrm{I}_{\mathrm{fix}}(j)$ is closed under the operations of $\mathcal{M}$. It is also clear by the definition of $\mathrm{I}_{\mathrm{fix}}(j)$ and the assumption that $j$ moves some element of $\mathcal{M}$ that $\mathrm{I}_{\text {fix }}(j)$ is a proper cut of $\mathcal{M}$. Hence $\mathrm{I}_{\text {fix }}(j) \models \mathrm{I} \Delta_{0}+\mathrm{B} \Sigma_{1}$ by Theorem 2.2 .

It remains to show that Exp holds in $\mathrm{I}_{\text {fix }}(j)$. First we will show:

(*) If $a \in \mathrm{I}_{\mathrm{fix}}(j)$ and $2^{a}$ is defined in $\mathcal{M}$, then $2^{a} \in \mathrm{I}_{\mathrm{fix}}(j)$.

To establish $(*)$, suppose $\mathcal{M} \models b<2^{a}$. Then $\mathcal{M} \models b=\sum_{i<c} 2^{s_{i}}$, with $c \leq a$ and $s_{0}<\cdots<s_{c-1}<a$. Therefore $j(c)=c$ and $j\left(s_{i}\right)=s_{i}$ for each $i<c$, because $a \in \mathrm{I}_{\mathrm{fix}}(j)$. So we have some element $b^{\prime} \in j(M)$ such that:

$$
j(\mathcal{M}) \models j(b)=\sum_{i<j(c)} 2^{j\left(s_{i}\right)}=\sum_{i<c} 2^{s_{i}}=b^{\prime} .
$$

But $j(\mathcal{M}) \prec_{\Delta_{0}} \mathcal{M}$ by assumption, and therefore the $j(\mathcal{M})$-binary representation of each element of $j(\mathcal{M})$ coincides with the $\mathcal{M}$-binary representation 
of the same element since for a sequence $s=\left\langle s_{i}: i\langle c\rangle\right.$ in $j(\mathcal{M})$, where $c$ might be nonstandard, the statement $x=\sum_{i<c} 2^{s_{i}}$ is well-known to be expressible in $j(\mathcal{M})$ by a $\Delta_{0}$-formula $\delta(x, s, p)$ (where $p$ is some sufficiently large parameter). This makes it clear that $b^{\prime}=b$. Therefore $j(b)=b$ for each $b<2^{a}$; which in turn implies that $2^{a} \in \mathrm{I}_{\mathrm{fix}}(j)$.

In light of $(*)$, the proof that Exp holds in $\mathcal{M}$ will be complete once we demonstrate that for all $a \in \mathrm{I}_{\text {fix }}(j), 2^{a}$ is defined in $\mathcal{M}$. Indeed, we will establish the slightly stronger result $(* *)$ below:

$(* *) \mathrm{I}_{\text {fix }}(j) \subsetneq J$, where $J:=\left\{x \in M: \mathcal{M} \models \exists y\left(2^{x}=y\right)\right\}$.

In order to verify $(* *)$, first let $P:=\left\{y \in M: \mathcal{M} \models \exists x\left(2^{x}=y\right)\right\}$, and note that:

(1) $P$ is unbounded in $\mathcal{M}$,

since otherwise by putting the fact that the graph of the exponential function is $\Delta_{0}$-definable in $\mathcal{M}$ (Theorem 2.1) together with the veracity of $\Delta_{0}$-Max in $\mathcal{M}$ (Theorem 2.3(d)), there would have to be a last power of 2 in $\mathcal{M}$, which is impossible. Next, note that if $(* *)$ fails, then:

(2) $J \subseteq \mathrm{I}_{\text {fix }}(j)$,

because $J$ is an initial segment of $\mathcal{M}$ by Theorem 2.1(b). By putting (2) together with $(*)$ we obtain:

(3) $P \subseteq \mathrm{I}_{\text {fix }}(j)$.

But since $j$ is assumed to be nontrivial, there is some $c \in M$ such that $\mathrm{I}_{\text {fix }}(j)<c$, and so by (3) $P$ is bounded above by $c$, which contradicts (1), and thereby concludes the proof of $(* *)$.

Theorem 3.3 below fine-tunes a result of Hájek \& Pudlák [12, Thm. 11]. Their result is the special case of Corollary 3.3.1 when $\mathcal{M}$ and $\mathcal{N}$, as well as the cut $I$, are all assumed to be models of PA.

3.3. Theorem. Suppose $\mathcal{M}$ and $\mathcal{N}$ are countable nonstandard models of $\mathrm{I} \Sigma_{1}$ with $c \in M$ and $a, b \in N$. Furthermore, suppose $I$ is a proper cut shared by $\mathcal{M}$ and $\mathcal{N}$ such that I is closed under exponentiation. The following are equivalent:

(1) There is a proper initial embedding $j: \mathcal{M} \rightarrow \mathcal{N}$ such that $j(c)=a$, $j(M)<b$ and $j(i)=i$ for all $i \in I$.

(2) $\operatorname{SSy}_{I}(\mathcal{M})=\operatorname{SSy}_{I}(\mathcal{N})$, and for all $i \in I$ and all $\Delta_{0}$-formulae $\delta(x, y, z)$, if $\mathcal{M} \models \exists z \delta(i, c, z)$, then $\mathcal{N} \models \exists z<b \delta(i, a, z)$.

Proof. $(1) \Rightarrow(2)$ is easy and is left to the reader so we will concentrate on $(2) \Rightarrow(1)$. Assume $(2)$ and fix an enumeration $\left(c_{k}: k<\omega\right)$ of $M$; and an 
enumeration $\left(d_{k}: k<\omega\right)$ of $N$ in which each element of $N$ occurs infinitely often. The proof of (1) will be complete by setting $j\left(u_{k}\right)=v_{k}$ once we have $\left(u_{k}: k<\omega\right)$ and $\left(v_{k}: k<\omega\right)$ that satisfy the following four conditions:

(I) $M=\left\{u_{k}: k<\omega\right\}$.

(II) $\left\{v_{k}: k<\omega\right\}$ is an initial segment of $\mathcal{N}$, and each $v_{k}<b$.

(III) $u_{0}=a$ and $v_{0}=c$.

(IV) For each positive $n<\omega$, each $i \in I$, and each $\Delta_{0}$-formula $\delta(x, \mathbf{y}, z)$, where $\mathbf{y}=\left(y_{r}: r<n\right)$, the following holds for $\mathbf{u}=\left(u_{r}: r<n\right)$, and $\mathbf{v}=$ $\left(v_{r}: r<n\right):$

$$
\mathcal{M} \models \exists z \delta(i, \mathbf{u}, z) \Longrightarrow \mathcal{N} \models \exists z<b \delta(i, \mathbf{v}, z) .
$$

We will define finite tuples $\mathbf{u}_{m}=\left(u_{r}: r<n_{m}\right)$ and, $\mathbf{v}_{m}=\left(v_{r}: r<n_{m}\right)$ from $M$ (and of the same length) by recursion on $m$ so that the following condition is maintained through the recursion for all $m<\omega$ :

$\left(*_{m}\right)$ If $\mathcal{M} \models \exists z \delta\left(i, \mathbf{u}_{m}, z\right)$, then $\mathcal{N} \models \exists z<b \delta\left(i, \mathbf{v}_{m}, z\right)$, for all $i \in I$, and each $\delta(x, \mathbf{y}, z) \in \Delta_{0}$, where $\mathbf{y}=\left(y_{r}: r<n_{m}\right)$.

For $m=0$, we set $\mathbf{u}_{0}=(a)$ and $\mathbf{v}_{0}=(c)$, so $n_{0}=1$. By (2) this choice of $\mathbf{u}_{0}$ and $\mathbf{v}_{0}$ satisfies $\left(*_{0}\right)$. Let $\left\langle\delta_{r}: r \in M\right\rangle$ be a canonical enumeration within $\mathcal{M}$ of all $\Delta_{0}$-formulae (e.g., as in [13, Ch. 1]). For $m \geq 0$, we may assume that there are $\mathbf{u}_{m}$ and $\mathbf{v}_{m}$ satisfying $\left(*_{m}\right)$. In order to construct $\mathbf{u}_{m+1}$ and $\mathbf{v}_{m+1}$ we distinguish between the case $m=2 k$ (the $k$-th 'forth' stage) and the case $m=2 k+1$ (the $k$-th 'back' stage) as described below.

CASE $m=2 k$. In this case, if $c_{k}$ is already among the elements listed in $\mathbf{u}_{m}$ we have nothing to do, i.e., in this case $\mathbf{u}_{m+1}=\mathbf{u}_{m}$ and $\mathbf{v}_{m+1}=\mathbf{v}_{m}$. Otherwise, consider:

$$
H:=\left\{\langle r, i\rangle \in I: \mathcal{M} \models \exists z \operatorname{Sat}_{\Delta_{0}}\left(\delta_{r}\left(i, \mathbf{u}_{m}, c_{k}, z\right)\right)\right\} .
$$

$H$ is the intersection of a $\Sigma_{1}$-definable subset of $\mathcal{M}$ with $I$, so $H \in \operatorname{SSy}_{I}(\mathcal{M})=$ $\operatorname{SSy}_{I}(\mathcal{N})$. Therefore we can choose $h$ in $\mathcal{M}$ and $h^{\prime}$ in $\mathcal{N}$ such that:

$$
H=I \cap\left(h_{E}\right)^{\mathcal{M}}=I \cap\left(h_{E}^{\prime}\right)^{\mathcal{N}} .
$$

For each $p \in M$ and $q \in N$ define:

$$
H_{p}:=\left(h_{E} \cap \underline{p}\right)^{\mathcal{M}} \text { and } H_{q}^{\prime}:=\left(h_{E}^{\prime} \cap \underline{q}\right)^{\mathcal{N}} .
$$


Choose $h_{p} \in M$ and $h_{q}^{\prime} \in N$ such that $H_{p}$ is coded by $h_{p}$ in $\mathcal{M}$, and $H_{q}^{\prime}$ is coded by $h_{q}^{\prime}$ in $\mathcal{N}$. In light of the assumption that $I$ is closed under exponentiation, we have:

(i) $h_{s}=h_{s}^{\prime} \in I$ for each $s \in I$.

On the other hand, by definition:

(ii) $s \in I \Rightarrow \mathcal{M} \models \forall\langle r, i\rangle \in h_{s} \exists z \operatorname{Sat}_{\Delta_{0}}\left(\delta_{r}\left(i, \mathbf{u}_{m}, c_{k}, z\right)\right)$.

Putting (ii) together with $\Sigma_{1}$-Collection in $\mathcal{M}$ yields:

(iii) $s \in I \Rightarrow \mathcal{M} \models \exists t \forall\langle r, i\rangle \in h_{s} \exists z<t \operatorname{Sat}_{\Delta_{0}}\left(\delta_{r}\left(i, \mathbf{u}_{m}, c_{k}, z\right)\right)$.

By quantifying out $c_{k}$ in (iii) we obtain:

(iv) $s \in I \Rightarrow \mathcal{M} \models \overbrace{\exists x \exists t \forall\langle r, i\rangle \in h_{s} \exists z<t \operatorname{Sat}_{\Delta_{0}}\left(\delta_{r}\left(i, \mathbf{u}_{m}, x, z\right)\right)}^{\varphi\left(h_{s}, \mathbf{u}\right)}$.

Note that $\varphi\left(h_{s}, \mathbf{u}_{m}\right)$ can be written as a $\Sigma_{1}$-formula. Therefore by coupling our inductive hypothesis $\left(*_{m}\right)$ with $(i)$ and $(i i i)$ we conclude:

$(v) s \in I \Rightarrow \mathcal{N} \models \exists x, t<b \forall\langle r, i\rangle \in h_{s}^{\prime} \exists z<t \operatorname{Sat}_{\Delta_{0}}\left(\delta_{r}\left(i, \mathbf{v}_{m}, x, z\right)\right)$.

Finally, by $(v)$ together and $\Sigma_{1}$-Overspill in $\mathcal{N}$ there exists $p>I$ such that:

(vi) $\mathcal{N} \models \exists x, t<b \forall\langle r, i\rangle \in h_{p}^{\prime} \exists z<t \operatorname{Sat}_{\Delta_{0}}\left(\delta_{r}\left(i, \mathbf{v}_{m}, x, z\right)\right)$.

Let $d$ be a witness in $\mathcal{N}$ to the $\exists x$ assertion in $(v i)$, and let $\mathbf{u}_{m+1}=\left(\mathbf{u}_{m}, c_{k}\right)$ and $\mathbf{v}_{m+1}=\left(\mathbf{v}_{n}, d\right)$. It is easy to see using $(v i)$ that $\left(*_{m+1}\right)$ holds with these choices of $\mathbf{u}_{m+1}$ and $\mathbf{v}_{m+1}$.

CASE $m=2 k+1$. If $d_{k}>\max \left(\mathbf{v}_{m}\right)$ we do nothing, i.e., we define $\mathbf{u}_{m+1}:=\mathbf{u}_{m}$ and $\mathbf{v}_{m+1}:=\mathbf{v}_{m}$. Otherwise, let:

$$
L=\left\{\langle r, i\rangle \in I: \mathcal{N} \models \forall z \quad\left(\operatorname{Sat}_{\Delta_{0}}\left(\delta_{r}\left(i, \mathbf{v}_{m}, d_{k}, z\right) \rightarrow b \leq z\right)\right\} .\right.
$$

Since $L$ is the intersection of a $\Pi_{1}$-definable subset of $\mathcal{N}$ with $I, L \in$ $\operatorname{SSy}_{I}(\mathcal{N})=\operatorname{SSy}_{I}(\mathcal{M})$. Therefore we can choose $l$ in $\mathcal{M}$ and $l^{\prime}$ in $\mathcal{N}$ such that:

$$
L=I \cap\left(l_{E}\right)^{\mathcal{M}}=I \cap\left(l_{E}^{\prime}\right)^{\mathcal{N}} .
$$

For each $p \in M$ and $q \in N$ define:

$$
L_{p}:=\left(l_{E} \cap \underline{p}\right)^{\mathcal{M}} \text { and } L_{q}^{\prime}:=\left(l_{E}^{\prime} \cap \underline{q}\right)^{\mathcal{N}} .
$$

Let $l_{p} \in M$ and $l_{q}^{\prime} \in N$ such that $L_{p}$ is coded by $l_{p}$ in $\mathcal{M}$, and $L_{q}^{\prime}$ is coded by $l_{q}^{\prime}$ in $\mathcal{N}$. The closure of $I$ under exponentiation makes it clear that:

(vii) $l_{s}=l_{s}^{\prime} \in I$ for each $s \in I$. 
We claim that for every $s \in I$ the following holds:

(viii) $\mathcal{M} \models \exists x \leq \max \left(\mathbf{u}_{m}\right) \forall\langle r, i\rangle \in l_{s} \forall z \neg \operatorname{Sat}_{\Delta_{0}}\left(\delta_{r}\left(i, \mathbf{u}_{m}, x, z\right)\right.$ ).

Suppose not, then for some $s \in I$ :

$(i x) \mathcal{M} \models \forall x \leq \max \left(\mathbf{u}_{m}\right) \exists\langle r, i\rangle \in l_{s} \exists z \operatorname{Sat}_{\Delta_{0}}\left(\delta_{r}\left(i, \mathbf{u}_{m}, x, z\right)\right)$.

Thanks to $\Sigma_{1}$-collection in $\mathcal{M}$ and (viii) we obtain:

$(x) \mathcal{M} \models \exists t \forall x \leq \max \left(\mathbf{u}_{m}\right) \exists\langle r, i\rangle \in l_{s} \exists z<t \operatorname{Sat}_{\Delta_{0}}\left(\delta_{r}\left(i, \mathbf{u}_{m}, x, z\right)\right)$.

So by our inductive assumption $\left(*_{m}\right)$ and $(x)$ we have:

$(x i) \mathcal{N} \models \exists t<b \forall x \leq \max \left(\mathbf{v}_{m}\right) \exists\langle r, i\rangle \in l_{s} \exists z<t \operatorname{Sat}_{\Delta_{0}}\left(\delta_{r}\left(i, \mathbf{v}_{m}, x, z\right)\right)$.

In particular, by choosing $x=d_{k}$ we obtain:

$(x i i) \mathcal{N} \models \exists\langle r, i\rangle<b \exists z<b\left(\langle r, i\rangle \in l_{s} \wedge \operatorname{Sat}_{\Delta_{0}}\left(\delta_{r}\left(i, \mathbf{v}_{m}, d_{k}, z\right)\right)\right)$,

which contradicts the definition of $l_{s}$.

By $\Pi_{1}$-Overspill in $\mathcal{M}$ there is some $q \in M \backslash I$ such that:

(xiii) $\mathcal{M} \models \exists x \leq \max \left(\mathbf{u}_{m}\right) \forall\langle r, i\rangle \in l_{q} \forall z \neg \operatorname{Sat}_{\Delta_{0}}\left(\delta_{r}, \mathbf{u}_{m}, x, z\right)$.

Let $c$ be a witness in $\mathcal{M}$ to the $\exists x$ assertion in (xiii), and let $\mathbf{u}_{m+1}=\left(\mathbf{u}_{m}, c\right)$ and $\mathbf{v}_{m+1}=\left(\mathbf{v}_{m}, d_{k}\right)$. It is easy to see using (xiii) that $\left(*_{m+1}\right)$ holds with these choices of $\mathbf{u}_{m+1}$ and $\mathbf{v}_{m+1}$.

This concludes the recursive construction of $\left(u_{k}: k \in \omega\right)$ and $\left(v_{k}: k \in \omega\right)$ satisfying properties (I) through (IV).

3.3.1. Corollary. Let $\mathcal{M}$ and $\mathcal{N}$ be countable nonstandard models of $\mathrm{I} \Sigma_{1}$, and $I$ be a proper cut shared by $\mathcal{M}$ and $\mathcal{N}$ that is closed under exponentiation. The following are equivalent:

(1) There is a proper initial embedding $j$ of $\mathcal{M}$ into $\mathcal{N}$ such that $j(i)=i$ for all $i \in I$.

(2) $\operatorname{Th}_{\Sigma_{1}}(\mathcal{M}, i)_{i \in I} \subseteq \operatorname{Th}_{\Sigma_{1}}(\mathcal{N}, i)_{i \in I}$ and $\operatorname{SSy}_{I}(\mathcal{M})=\operatorname{SSy}_{I}(\mathcal{N})$.

Proof. $(1) \Rightarrow(2)$ is again the easy direction. To show that $(2) \Rightarrow(1)$, by Theorem 3.3 it suffices to show (2) implies that there are $c \in M$ and $a, b \in N$ such that for all $i \in I$ and $\Delta_{0}$-formulae $\delta(x, y, z)$, if $\mathcal{M} \models \exists z \delta(i, c, z)$, then $\mathcal{N} \models \exists z<b \delta(i, a, z)$. Let $a=c=0$. We need to show that for some $b \in N$ such that for all $i \in I$ and $\Delta_{0}$-formulae $\delta(x, z)$, if $\mathcal{M} \models \exists z \delta(i, z)$, then $\mathcal{N} \models \exists z<b \delta(i, z)$. Let $\left\langle\delta_{i}: i \in N\right\rangle$ be a canonical enumeration within $\mathcal{N}$ of all $\Delta_{0}$-formulae, and for $s \in N$ let $\varphi(s)$ be the following statement:

$$
\exists y_{s} \forall\langle r, i\rangle<s\left[\exists x \operatorname{Sat}_{\Delta_{0}}\left(\delta_{r}(i, x)\right) \rightarrow \exists x<y_{s} \operatorname{Sat}_{\Delta_{0}}\left(\delta_{r}(i, x)\right)\right] .
$$


By Strong $\Sigma_{1}$-collection in $\mathcal{N}, \varphi(s)$ holds in $\mathcal{N}$ for any $s \in N$. In particular, if $s \in N \backslash I$ then $y_{s}$ serves as our desired $b$.

3.3.2. Remark. For any element $a_{0}$ of $\mathcal{M} \models \mathrm{I} \Sigma_{1}$, let $\left(a_{n}: n<\omega\right)$ be given by $\mathcal{M} \models a_{n+1}=2^{a_{n}}$; and consider:

$$
I:=\left\{m \in M: \exists n \in \omega \text { such that } m<a_{n}\right\} .
$$

$I$ is by design closed under exponentiation; it also forms a proper cut in $\mathcal{M}$ (thanks to the totality of the superexponential function in $\mathcal{M}$ ). This makes it clear that the $(2) \Rightarrow(1)$ direction of Corollary 3.3.1 implies the $(1) \Rightarrow(2)$ direction of Theorem 2.9 .

3.4. Theorem. For any countable nonstandard model $\mathcal{M}$ of $\mathrm{PA}$ there is a composition preserving embedding $j \longmapsto \widehat{j}$ of $\operatorname{PISE}(\mathbb{Q})$ into $\operatorname{PISE}(\mathcal{M})$, where $\mathbb{Q}$ is the ordered set of rationals. Moreover, if $j$ is contractive, then so is $\widehat{j}$.

Proof. Given a countable model $\mathcal{M}$ of PA, choose $\mathcal{A}$ be the collection of subsets of $M$ that are parametrically definable in $\mathcal{M}$, and let $\mathcal{N}_{\mathbb{Q}}$ be an elementary end extension of $\mathcal{M}$ as in Theorem 2.6. Since $\mathcal{M}$ and $\mathcal{N}_{\mathbb{Q}}$ share the same standard system and the same first order theory, Theorem 2.8 assures us that there is a proper initial embedding $k: \mathcal{M} \rightarrow \mathcal{N}_{\mathbb{Q}}$ such that $M \subsetneq k(M)$. Let $M^{*}=k(M)$. By part (c) of Theorem 2.6 we may choose $c_{q_{0}} \in M^{*} \backslash M$. Let $j \in \operatorname{PISE}(\mathbb{Q})$ such that $j(\mathbb{Q})<q_{0}$. By parts $(\mathrm{d})$ and $(\mathrm{g})$ of Theorem 2.6:

$$
\widehat{j} \in \operatorname{PISE}\left(\mathcal{N}_{\mathbb{Q}}\right) \text { and } \widehat{j}\left(N_{\mathbb{Q}}\right)<c_{q_{0}} \text {. }
$$

Therefore $\widehat{j}\left(M^{*}\right)<c_{q_{0}} \in M^{*}$. Let $\widehat{j}_{M^{*}}$ be the restriction of $\widehat{j}$ to $M^{*}$. Then $\widehat{j}_{M^{*}} \in \operatorname{PISE}\left(\mathcal{M}^{*}\right)$ and the desired embedding of $\operatorname{PISE}(\mathbb{Q})$ into $\operatorname{PISE}(\mathcal{M})$ is $j \mapsto k^{-1} \circ \widehat{j}_{M^{*}} \circ k$.

3.4.1. Remark. It is easy to see, using Cantor's theorem asserting that any countable dense linear order without endpoints is isomorphic to $\mathbb{Q}$, that $\mathbb{Q}$ carries a proper initial self-embedding that is contractive.

3.4.2. Corollary. Every countable nonstandard model of PA carries a contractive proper initial self-embedding.

Proof. Put Theorem 3.4 together with Remark 3.4.1.

3.4.3. Proposition. For every countable linear order $\mathbb{L}$, there is a composition preserving embedding $j \mapsto \widehat{j}$ of $\mathrm{SE}(\mathbb{L})$ into $\mathrm{SE}(\mathbb{Q})$. Moreover: 
(a) $\hat{j} \in \operatorname{Aut}(\mathbb{Q})$ iff $j \in \operatorname{Aut}(\mathbb{L})$.

(b) $\hat{j} \in \operatorname{ISE}(\mathbb{Q})$ iff $j \in \operatorname{ISE}(\mathbb{L})$.

(c) $\widehat{j} \in \operatorname{PISE}(\mathbb{Q})$ iff $j \in \operatorname{PISE}(\mathbb{L})$.

Proof. Given a linear order $\mathbb{L}$, let $\mathbb{L} \times \mathbb{Q}$ be the lexicographic product of $\mathbb{L}$ and $\mathbb{Q}$ (intuitively $\mathbb{L} \times \mathbb{Q}$ is the result of replacing each point in $\mathbb{L}$ by a copy of $\mathbb{Q}) . \mathbb{L} \times \mathbb{Q}$ is clearly a countable dense linear order with no end points. Therefore when $\mathbb{L}$ is countable, $\mathbb{L} \times \mathbb{Q}$ is isomorphic to $\mathbb{Q}$ by Cantor's theorem mentioned in Remark 3.4.1. So it suffices to find a composition preserving embedding of $\operatorname{SE}(\mathbb{L})$ into $\operatorname{SE}(\mathbb{L} \times \mathbb{Q})$ that satisfies (a), (b), and (c). Given $j \in \mathrm{SE}(\mathbb{L})$, let $\widehat{j}: \mathbb{L} \times \mathbb{Q} \rightarrow \mathbb{L} \times \mathbb{Q}$ by $\widehat{j}(l, q)=(j(l), q)$. A routine reasoning shows that $\hat{j} \in \mathrm{SE}(\mathbb{L} \times \mathbb{Q})$, and the embedding $j \mapsto \widehat{j}$ is composition preserving. Properties (a), (b), and (c) are equally easy to verify.

3.4.4. Remark. Let $\mathcal{M}=(M,<, \cdots)$ be a linearly ordered structure. $\mathrm{SE}(\mathcal{M})$ is a sub-semigroup of $\mathrm{SE}(M,<)$, therefore by Proposition 3.4.3 $\operatorname{SE}(\mathcal{M})$ is embeddable into $\operatorname{SE}(\mathbb{Q}) ; \operatorname{Aut}(\mathcal{M})$ is embeddable in $\operatorname{Aut}(\mathbb{Q}) ; \operatorname{ISE}(\mathcal{M})$ is embeddable in $\operatorname{ISE}(\mathbb{Q})$; and $\operatorname{PISE}(\mathcal{M})$ is embeddable in $\operatorname{PISE}(\mathbb{Q})$.

\section{THE LONGEST INITIAL SEGMENT OF FIXED POINTS}

In this section we establish the first principal result of this paper (Theorem 4.1) by an elaboration of the back-and-forth proof of Theorem 3.3. The $(2) \Rightarrow(3)$ direction of Theorem 4.1 fine-tunes the $(1) \Rightarrow(2)$ direction of Theorem 2.9, since as pointed out in Remark 3.3.2 proper cuts closed under exponentiation can be found arbitrarily high in every nonstandard model of $\mathrm{I} \Sigma_{1}$

4.1. Theorem. Suppose $I$ is a proper initial segment of a countable nonstandard model $\mathcal{M}$ of $\mathrm{I} \Sigma_{1}$. The following are equivalent:

(1) $I=\mathrm{I}_{\mathrm{fix}}(j)$ for some self-embedding $j$ of $\mathcal{M}$.

(2) $I$ is closed under exponentiation.

(3) $I=\mathrm{I}_{\mathrm{fix}}(j)$ for some proper initial self-embedding $j$ of $\mathcal{M}$.

Proof. $(1) \Rightarrow(2)$ follows immediately from Lemma 3.1.1 and Theorem 3.2; and $(3) \Rightarrow(1)$ is trivial; so it suffices to establish $(2) \Rightarrow(3)$. By the proof of Corollary 3.3.1 we can let $a=c=0$, and let $b$ be a large enough element of $\mathcal{M}$ such that for all $i \in I$ and all $\Delta_{0}$-formulae $\delta(x, y, z)$ we have: 


$$
\mathcal{M} \models \exists z \delta(i, c, z) \rightarrow \exists z<b \delta(i, a, z) .
$$

Assume (2). In order to produce the desired embedding $j$ satisfying (3) we will elaborate the proof of Theorem 3.3 by adding a third layer of recursion to the proof of Theorem 3.3. More specifically, at stage $m=3 k$ we will do the same as stage $m=2 k$ of the proof of Theorem 3.3, and at stage $m=3 k+1$ we will do the same as stage $m=2 k+1$ of the proof of Theorem 3.3. In order to describe the construction for stages $m$ of the form $3 k+2$, we first establish the following lemma:

4.1.1. Lemma Suppose $\mathbf{u}$ and $\mathbf{v}$ are finite tuples of the same length from $\mathcal{M}$ that satisfy:

(I) $\mathcal{M} \models \exists z \delta(i, \mathbf{u}, z) \rightarrow \exists z<b \delta(i, \mathbf{v}, z)$ for any $i \in I$ and any $\delta(x, \mathbf{y}, z) \in$ $\Delta_{0}$

Then for any $d \in M \backslash I$ there are distinct $u, v \in M$ such that $u<d$ and:

(II) $\mathcal{M} \models \exists z \delta(i, \mathbf{u}, u, z) \rightarrow \exists z<b \delta(i, \mathbf{v}, v, z)$ for any $i \in I$ and any $\delta(x, \mathbf{y}, w, z) \in \Delta_{0}$.

Proof. Assume (I) holds and suppose $d \in M \backslash I$. Let $\left\langle\delta_{i}: i \in M\right\rangle$ be a canonical enumeration within $\mathcal{M}$ of all $\Delta_{0}$-formulae. For $s \in I$ and $x<d$, let:

$$
H_{s, x}:=\left\{\langle r, i\rangle<s: \exists z \operatorname{Sat}_{\Delta_{0}}\left(\delta_{r}(i, \mathbf{u}, x, z)\right)\right\} .
$$

Then define $f_{s}: \underline{d} \rightarrow \underline{2^{s+1}}$ in $\mathcal{M}$ for $x<d$ via:

$$
f_{s}(x)=\sum_{\langle r, i\rangle \in H_{s, x}} 2^{\langle r, i\rangle}
$$

Note that $f_{s}(x) \leq \sum_{k<s} 2^{k}=2^{s+1}-1$, which coupled with the closure of $I$ under exponentiation implies:

$$
d>2^{s+1}>f_{s}(x)
$$

On the other hand, for each $x, f_{s}(x)$ is $\Sigma_{1}$-minimal (in parameters $x$ and $s$ ), and therefore the graph of $f_{s}$ is $\Delta_{0}\left(\Sigma_{1}\right)$-definable in $\mathcal{M}$, so by $\Delta_{0}\left(\Sigma_{1}\right)$-PHP, $f_{s}$ is not one-to-one, and we may therefore choose distinct $u, u^{\prime}<d$ such that $f_{s}(u)=f_{s}\left(u^{\prime}\right)$. Let $\varphi(s)$ be the formula:

$$
\exists u, u^{\prime}<d\left(\left(u \neq u^{\prime}\right) \wedge \theta\left(s, u, u^{\prime}\right)\right)
$$

where $\theta\left(s, u, u^{\prime}\right)$ is: 


$$
\forall\langle r, i\rangle<s\left[\exists z \operatorname{Sat}_{\Delta_{0}}\left(\delta_{r}(i, \mathbf{u}, u, z)\right) \leftrightarrow \exists z \operatorname{Sat}_{\Delta_{0}}\left(\delta_{r}\left(i, \mathbf{u}, u^{\prime}, z\right)\right)\right] .
$$

The definition of $f_{s}$ makes it evident that $\mathcal{M} \models \varphi(s)$ for each $s \in I$. Since $\varphi(s)$ is a $\Delta_{0}\left(\Sigma_{1}\right)$ statement, by $\Delta_{0}\left(\Sigma_{1}\right)$-Overspill in $\mathcal{M}$ there is some $p \in$ $M \backslash I$ such that $\mathcal{M} \models \varphi(p)$. Therefore there are distinct $u, u^{\prime}<d$ such that for each $i \in I$ and each $\Delta_{0}$-formula $\delta$ we have:

(i) $\mathcal{M} \models \exists z \delta(i, \mathbf{u}, u, z) \leftrightarrow \exists z \delta\left(i, \mathbf{u}, u^{\prime}, z\right)$.

On the other hand, by the proof of the 'forth' direction (the $m=2 k$ case) of Theorem 3.3, we can find distinct $w$ and $w^{\prime}$ such that the following holds for each $\Delta_{0}$-formula $\delta$ :

(ii) $\mathcal{M} \models \exists z \delta\left(i, \mathbf{u}, u, u^{\prime}, z\right) \rightarrow \exists z<b \delta\left(i, \mathbf{v}, w, w^{\prime}, z\right)$.

Since at least one of the two statements $\left\{u \neq w, u \neq w^{\prime}\right\}$ is true, we can choose $v \in\left\{w, w^{\prime}\right\}$ such that $u \neq v$. It is easy to see using $(i)$ and (ii) that this choice of $u$ and $v$ satisfy (II).

Lemma 4.1.1

Fix a sequence $\left(d_{k}: k \in \omega\right)$ that is downward cofinal in $M \backslash I$. Suppose $m=3 k+2$ and we have $\mathbf{u}_{m}$ and $\mathbf{v}_{m}$ satisfying condition $\left(*_{m}\right)$ of the proof of Theorem 3.3 for $\mathcal{N}:=\mathcal{M}$. Apply Lemma 4.1 .1 with $\mathbf{u}:=\mathbf{u}_{m}, \mathbf{v}:=\mathbf{v}_{m}$, and $d:=d_{k}$ to get hold of $u$ and $v$ satisfying (II) of Lemma 4.1.1; and then we define $\mathbf{u}_{m+1}:=\left(\mathbf{u}_{m}, u\right)$ and $\mathbf{v}_{m+1}:=\left(\mathbf{v}_{m}, v\right)$. This makes it clear that the proper initial self-embedding $j$ of $\mathcal{M}$ defined by $j\left(u_{k}\right)=v_{k}$ fixes each $i \in I$ but moves elements arbitrarily low in $M \backslash I$.

4.1.2. Remark. By Remark 3.3.2 there are unboundedly many cuts in a nonstandard model of $\mathrm{I} \Sigma_{1}$ that are closed under exponentiation. Therefore Theorem 3.3 is a strengthening of the $(1) \Rightarrow(2)$ direction of Theorem 2.9. Also, it is easy to see (using an overspill argument) that in nonstandard models of $\mathrm{I} \Delta_{0}$ cuts that are closed under exponentiation can be found arbitrarily low in the nonstandard part of $\mathcal{M}$.

\section{FIXED POINT SETS THAT ARE INITIAL SEGMENTS}

This section is devoted to the second main result of this paper (Theorem 5.1). See also Remark 5.1.1.

5.1. Theorem. Suppose $I$ is a proper initial segment of a countable nonstandard model $\mathcal{M}$ of $\mathrm{I}_{1}$. The following are equivalent:

(1) $I=\operatorname{Fix}(j)$ for some self-embedding $j$ of $\mathcal{M}$.

(2) $I$ is a strong cut of $\mathcal{M}$, and $I \prec_{\Sigma_{1}} \mathcal{M}$. 
(3) $I=\operatorname{Fix}(j)$ for some proper initial self-embedding $j$ of $\mathcal{M}$.

Proof. Since $(3) \Rightarrow(1)$ is trivial, it suffices to show (1) $\Rightarrow(2)$ and (2) $\Rightarrow(3)$.

To verify $(1) \Rightarrow(2)$, suppose (1) holds and let $\tilde{f} \in M$ code an $\mathcal{M}$-finite function $f$ whose domain includes $I$. It is easy to see that $\tilde{f} \notin I$. So if $\widetilde{g}:=j(\widetilde{f})$, then $\widetilde{g} \notin I$, and $\widetilde{f} \neq \widetilde{g}$. Therefore, in light of the assumption that $I=\operatorname{Fix}(j)$, if $g$ is the function that is coded by $\widetilde{g}$, then:

$$
\forall i \in I[f(i)=g(i) \Longleftrightarrow f(i) \in I] .
$$

We wish to find $s \in M \backslash I$ such that for all $i \in I, f(i) \notin I$ iff $s<f(i)$. Fix $d \in M$ such that $I<d$ and the interval $[0, d] \subseteq \operatorname{dom}(f) \cap \operatorname{dom}(g)$. Without loss of generality there is some $i_{0} \in I$ with $f\left(i_{0}\right) \notin I$. Consider the function $h(x)$ defined within $\mathcal{M}$ on the interval $\left[i_{0}, d\right]$ by:

$$
h(x):=\mu y \leq d[\exists z \leq x(y=f(z) \neq g(z)],
$$

where $\mu y \leq d$ is the modified least search operator, defined via the following:

$$
[z:=\mu y \leq d \varphi(y)]
$$

$[z$ is the first $y$ such that $\varphi(y)$, if $\exists y \leq d \varphi(y)$; else $z=d]$.

Note that if $i \in I$, then $h(i) \notin I$, and if $i_{0} \leq i \leq i^{\prime}$, then $h\left(i^{\prime}\right) \leq h(i)$. Moreover:

(i) The graph of $h$ is defined by a $\Delta_{0}$-formula $\varphi(x, y)$ with parameters $\tilde{f}, \widetilde{g}$, and $d$.

(ii) $i<h(i)$ for all $i \in I$ such that $i \geq i_{0}$.

Therefore, by putting $(i)$ together with $(i i)$ and $\Delta_{0}$-Overspill we may conclude that there is some $s \in M \backslash I$ such that $s<h(s)$ holds in $\mathcal{M}$. This shows that $s$ is the desired lower bound for elements of the form $f(i)$, where $i \in I$ and $f(i) \notin I$. This concludes the verification that $I$ is a strong cut of $\mathcal{M}$. On the other hand, since we are assuming that (1) holds, Theorem 3.1 assures that $I \prec_{\Sigma_{1}} \mathcal{M}$, so (2) holds.

To establish $(2) \Rightarrow(3)$, suppose (2) holds. We first note that by Theorem $2.5,\left(I, \operatorname{SSy}_{I}(\mathcal{M})\right) \models \mathrm{ACA}_{0}$. By Theorem 2.6 we can build $\mathcal{N}_{\mathbb{Q}} \succ$ end $I$ (where $\mathbb{Q}$ is the ordered set of rationals) such that:

(iii) $\operatorname{SSy}_{I}(\mathcal{M})=\operatorname{SSy}_{I}\left(\mathcal{N}_{\mathbb{Q}}\right)$, and

(iv) $\mathbb{Q}^{\prime}:=\left\{c_{q}: q \in \mathbb{Q}\right\}$ is an isomorphic copy of $\mathbb{Q}$ and is downward cofinal in $\mathcal{N}_{\mathbb{Q}} \backslash I$. 
On the other hand, since $I \prec_{\Sigma_{1}} \mathcal{M}$ we may infer that $\operatorname{Th}_{\Sigma_{1}}(\mathcal{M}, i)_{i \in I}=$ $\mathrm{Th}_{\Sigma_{1}}\left(\mathcal{N}_{\mathbb{Q}}, i\right)_{i \in I}$, which together with $(i i i)$ and Corollary 3.3.1 allows us to get hold of an initial embedding $k: \mathcal{M} \rightarrow \mathcal{N}_{\mathbb{Q}}$ such that $k$ pointwise fixes each $i \in I$. Let $M^{*}$ be the range of $k$. By $(i v)$ there is some $q_{0} \in \mathbb{Q}$ and $m_{0}^{*} \in M^{*}$ such that:

(v) $c_{q_{0}}<m_{0}^{*}$.

Let $j_{0}: \mathbb{Q} \rightarrow \mathbb{Q}$ be a proper initial self-embedding of $\mathbb{Q}$ whose range is bounded above by $q_{0}$. By Theorem 2.6 the range of the induced initial selfembedding $\widehat{j}_{0}$ of $\mathcal{N}_{\mathbb{Q}}$ is bounded above by $c_{q_{0}}$ and $\operatorname{Fix}\left(\widehat{j}_{0}\right)=I$. Coupled with $(v)$ this shows that $\widehat{j_{0}}\left(M^{*}\right) \subsetneq M^{*}$. So we can identify $\mathcal{M}$ with its isomorphic copy $\mathcal{M}^{*}$ to complete the proof; in other words the desired $j \in \operatorname{PISE}(\mathcal{M})$ such that $\operatorname{Fix}(j)=I$ is given by $j:=k^{-1} \widehat{j}_{0} k$.

5.1.1. Remark. For each $n \in \omega$, there is a countable model of I $\Sigma_{n}$ which does not carry a proper cut $I$ satisfying (2) of Theorem 5.1 . To see this, first note that (2) implies that $\mathcal{M} \models \operatorname{Con}\left(\mathrm{I} \Sigma_{n}\right)$ for each $n<\omega$ since PA holds in $I$ by Theorem $2.5, \operatorname{Con}\left(\mathrm{I} \Sigma_{n}\right)$ is a $\Pi_{1}$-statement, and it is well-known [14, Ex. 10.8] that $\operatorname{Con}\left(\mathrm{I} \Sigma_{n}\right)$ is provable in $\mathrm{I} \Sigma_{n+1}$ for each $n \in \omega$. On the other hand, $\operatorname{Con}\left(\mathrm{I} \Sigma_{n}\right)$ is unprovable in $\mathrm{I} \Sigma_{n}$ by Gödel's second incompleteness theorem, and therefore there is a countable nonstandard model $\mathcal{M}_{0}$ of $\mathrm{I} \Sigma_{n}+$ $\neg \operatorname{Con}\left(\mathrm{I} \Sigma_{n}\right)$. Such a model $\mathcal{M}_{0}$ has no cut that satisfies condition (2) of Theorem 5.1. However, if $\mathcal{M}$ is a countable nonstandard model of PA, then by using a variation of the proof of Tanaka's theorem in [8], for any $n \in \omega$ we can find a strong cut $I$ arbitrarily high in $\mathcal{M}$ such that $I \prec_{\Sigma_{n}} \mathcal{M}$. Tin Lok Wong has also pointed out to us that there are countable models $\mathcal{M}_{0}$ of $\mathrm{I} \Sigma_{1}$ in which there is no proper cut $I$ such that $I \prec_{\Sigma_{1}} \mathcal{M}_{0}$. Such a model $\mathcal{M}_{0}$ can be readily obtained by choosing $\mathcal{M}_{0}$ as $H^{1}(\mathcal{M})$, where $\mathcal{M} \models I \Sigma_{1}$ and $H^{1}(\mathcal{M})$ is defined as in [13, Ch. IV, Def. 1.32].

\section{MINIMAL FIXED POINTS}

In this section we establish our final principal result (Theorem 6.1). The proof of Theorem 6.1 is rather complex and based on several technical lemmas, which were inspired by, and can be seen as miniaturized analogues of Lemmas 8.6.4, 8.6.6, and 8.6.2 of [16] (which were originally established in the joint work of Kaye, Kossak, and Kotlarski [15]).

Recall from Theorem 3.1 that $K^{1}(\mathcal{M}) \subseteq \operatorname{Fix}(j)$ for every $j \in \operatorname{SE}(\mathcal{M})$, where $\mathcal{M} \models \mathrm{I} \Delta_{0}+$ Exp. It is also straightforward to modify the proof of the 
basic Friedman embedding theorem [14, Thm. 12.3] to show that if $\mathcal{M}$ is a countable nonstandard model of $\mathrm{I} \Sigma_{1}$, and $m \in M \backslash K^{1}(\mathcal{M})$, then there is some $j \in \operatorname{PISE}(\mathcal{M})$ such that $j(m) \neq m$. These results motivate the question whether every countable nonstandard model $\mathcal{M} \models \mathrm{I} \Sigma_{1}$ has a proper initial self-embedding that moves all elements of $M \backslash K^{1}(\mathcal{M})$. Theorem 6.1 provides a complete answer to this question.

6.1. Theorem. The following are equivalent for a countable nonstandard model $\mathcal{M}$ of $\mathrm{I} \Sigma_{1}$ :

(1) $\operatorname{Fix}(j)=K^{1}(\mathcal{M})$ for some self-embedding $j$ of $\mathcal{M}$.

(2) $\mathbb{N}$ is a strong cut of $\mathcal{M}$.

(3) $\operatorname{Fix}(j)=K^{1}(\mathcal{M})$ for some proper initial self-embedding $j$ of $\mathcal{M}$.

Proof. Since $(3) \Rightarrow(1)$ is trivial, it suffices to show that $(1) \Rightarrow(2)$, and $(2) \Rightarrow(3)$.

\section{Proof of $(1) \Rightarrow(2)$ of Theorem 6.1}

The proof is based on Lemma 6.1.1, 6.1.2, and 6.1.4 below.

6.1.1. Lemma. If $\mathbb{N}$ is not a strong cut of $\mathcal{M} \models \mathrm{I} \Delta_{0}$, then for any selfembedding $j$ of $\mathcal{M}$, the nonstandard fixed points of $j$ are downward cofinal in the nonstandard part of $\mathcal{M}$.

Proof. Suppose that $\mathbb{N}$ is not strong in $\mathcal{M}$. Then there is some function $f$ coded in $\mathcal{M}$ whose domain is of the form $\underline{c}$ for some nonstandard $c$, and such that $D:=\{f(n): n \in \mathbb{N}$ and $f(n) \in M \backslash \mathbb{N}\}$ is downward cofinal in the nonstandard part of $\mathcal{M}$. Let $j$ be a self-embedding of $\mathcal{M}$, and let $g:=j(f)$. We observe that for each standard number $n$ the statement $P(n)$ holds in $\mathcal{M}$, where:

$$
P(z):=\text { "For all } x, y<z, f(x)=y \text { iff } g(x)=y " .
$$

Since $P(z)$ is a $\Delta_{0}$-formula (with parameters $f$ and $g$ ), by $\Delta_{0}$-Overspill for any nonstandard $k \in M$ there is some nonstandard $c<k$ such that $P(c)$ holds in $\mathcal{M}$. So it suffices to show that there is a nonstandard fixed point below any such $c$. Going back to the set $D$ defined earlier, let $n_{0} \in \mathbb{N}$ such that $f\left(n_{0}\right)$ is nonstandard and $f\left(n_{0}\right)<c$. Note that $f\left(n_{0}\right)=g\left(n_{0}\right)$ since $P(c)$ holds in $\mathcal{M}$, therefore:

$$
j\left(f\left(n_{0}\right)\right)=j(f)\left(j\left(n_{0}\right)\right)=g\left(n_{0}\right)=f\left(n_{0}\right) .
$$


So $f\left(n_{0}\right)$ is the desired nonstandard fixed point of $j$ below $c$.

6.1.2. Lemma. Suppose $\mathbb{N}$ is not a strong cut of $\mathcal{M} \models I \Sigma_{1}$. Then for every element $a \in M$ and any self-embedding $j$ of $\mathcal{M}$ there is an element $b \in \operatorname{Fix}(j)$ such that:

$$
\operatorname{Th}_{\Sigma_{1}}(\mathcal{M}, a) \subseteq \operatorname{Th}_{\Sigma_{1}}(\mathcal{M}, b)
$$

Proof. Let $\left\langle\sigma_{i}(x): i \in M\right\rangle$ be a canonical enumeration within $\mathcal{M}$ of all $\Sigma_{1}$-formulae in one free variable $x$, with $\sigma_{i}(x)=\exists y \delta_{i}(x, y)$, where $\delta_{i}$ is a $\Delta_{0}$-formula in the sense of $\mathcal{M}$. Recall that $(x)_{i}$ refers to the $i$-th coordinate of the sequence canonically coded by $x$, and the graph of $(x)_{i}$ is $\Delta_{0}$-definable.

Given $a \in M$, for any $k \in M,\left\{i<k: \exists y \operatorname{Sat}_{\Delta_{0}}\left(\delta_{i}(a, y)\right)\right\}$ is coded by some $\mathcal{M}$-finite $s_{k}$ thanks to part (b) of Theorem 2.3 and the fact that Sat $_{\Delta_{0}} \in \Sigma_{1}^{\mathrm{I} \Sigma_{1}}$. Note that the mapping $k \mapsto s_{k}$ is $\Sigma_{1}$-definable in $(\mathcal{M}, a)$. This makes it clear that for any $k \in M$ there is $c_{k} \in M$ such that:

$$
\mathcal{M} \models c_{k}=\min \left\{m \in M: \operatorname{Sat}_{\Delta_{0}}\left(\bigwedge_{i E s_{k}} \delta_{i}\left((m)_{0},(m)_{i+1}\right)\right)\right\}
$$

We observe that:

(i) For each nonstandard $k \in M \quad \operatorname{Th}_{\Sigma_{1}}(\mathcal{M}, a) \subseteq \operatorname{Th}_{\Sigma_{1}}\left(\mathcal{M},\left(c_{k}\right)_{0}\right)$.

Fix a nonstandard $i \in M$ choose $d \in M$ with $(d)_{k}=\left(c_{k}\right)_{0}$ for all $k<i$. Note that $(d)_{n} \in K^{1}(\mathcal{M})$ for $n \in \omega$, and therefore $j\left((d)_{n}\right)=(d)_{n}$ for $n \in \omega$. On the other hand, if we let $e:=j(d)$, then for $n \in \omega$ :

$$
j\left((d)_{n}\right)=(j(d))_{j(n)}=(e)_{n} .
$$

This shows that $(d)_{n}=(e)_{n}$ for $n \in \omega$, so if we let:

$$
\varphi(x):=\forall i<x(d)_{i}=(e)_{i},
$$

then $\varphi(n)$ holds in $\mathcal{M}$ for each $n \in \omega$; hence by $\Delta_{0}$-Overspill there is some nonstandard $n^{*}$ below $i$ such that $(d)_{k}=(e)_{k}$ for all $k \leq n^{*}$. Therefore by Lemma 6.1.1 there is a nonstandard $k \in M$ that is below $n^{*}$ such that:

(ii) $(d)_{k}=(e)_{k}$ and $j(k)=k$.

Since $(d)_{k}=\left(c_{k}\right)_{0}$ by design, in light of $(i)$ the proof of our lemma will be complete once we observe that $(d)_{k} \in \operatorname{Fix}(j)$ since by $(i i)$ we have: 


$$
j\left((d)_{k}\right)=(j(d))_{j(k)}=(e)_{k}=(d)_{k}
$$

It is convenient to employ the notion of a partial recursive function of $\mathcal{M}$ in order to state the next lemma; this notion will also play a key role in the proof of $(2) \Rightarrow(3)$ of Theorem 6.1 .

6.1.3. Definition. A partial function $f$ from $M$ to $M$ is a partial recursive function of $\mathcal{M}$ iff the graph of $f$ is definable in $\mathcal{M}$ by a parameter-free $\Sigma_{1}$ formula; i.e., there is some $\Delta_{0}$-formula $\delta(x, y, z)$ such that for all elements $r$ and $s$ of $\mathcal{M}$ :

$$
f(r)=s \text { iff } \mathcal{M} \models \exists z \delta(r, s, z) .
$$

Given such an $f$, we will write $[f(x) \downarrow]$ as an abbreviation for $\exists y \exists z \delta(x, y, z)$, and $[f(x) \downarrow]^{<w}$ as an abbreviation for:

$$
\exists y, z<w \delta(x, y, z) .
$$

Note that a partial recursive function $f$ naturally induces for each positive $n \in \omega$ a partial function from $M^{n}$ to $M$, which we will also denote by $f$, via:

$$
f\left(a_{1}, \cdots, a_{n}\right):=f\left(\left\langle a_{1}, \cdots, a_{n}\right\rangle\right) .
$$

- We shall use $\mathcal{F}$ to denote the collection of all partial recursive functions of $\mathcal{M}$.

6.1.4. Lemma. If $\mathcal{M} \models \mathrm{I} \Delta_{0}$, then:

$$
K^{1}(\mathcal{M})=\{f(0): f \in \mathcal{F} \text { and } \mathcal{M} \models[f(0) \downarrow]\} .
$$

Proof. This is an immediate consequence of part (b) of Lemma 3.1.2.

With the above lemmas in place we are now ready to present the proof of $(1) \Rightarrow(2)$ by demonstrating its contrapositive. Suppose $\mathbb{N}$ is not a strong cut of $\mathcal{M}$. Consider the type $p(x)$ consisting of the $\Sigma_{1}$-formulae of the form $[f(0) \downarrow] \wedge x \neq f(0)$, as $f$ ranges over the partial recursive functions of $\mathcal{M}$. By Lemma 6.1.4 no element of $K^{1}(\mathcal{M})$ realizes $p(x)$, and yet $p(x)$ is realized by every element of $M \backslash K^{1}(\mathcal{M})$, and of course $M \backslash K^{1}(\mathcal{M}) \neq \varnothing$ $\left(\mathrm{B} \Sigma_{1}\right.$ holds in $\mathcal{M}$, but not in $K^{1}(\mathcal{M})$ by $n=0$ case of part (b) of Theorem 2.4). In particular, if $a$ is chosen as an element of $M \backslash K^{1}(\mathcal{M})$ then for $b \in M$, $\operatorname{Th}_{\Sigma_{1}}(\mathcal{M}, a) \subseteq \operatorname{Th}_{\Sigma_{1}}(\mathcal{M}, b)$ implies $b \notin K^{1}(\mathcal{M})$. Hence $K^{1}(\mathcal{M}) \neq \operatorname{Fix}(j)$ by Lemma 6.1.2. This concludes the proof of $(1) \Rightarrow(2)$ of Theorem 6.1. 


\section{Proof of $(2) \Rightarrow(3)$ of Theorem 6.1}

Assume (2). Since $\operatorname{Sat}_{\Sigma_{1}}$ has a $\Sigma_{1}$-description in $\mathcal{M}$ and strong $\Sigma_{1^{-}}$ collection holds in $\mathcal{M}$, there is a sufficiently large $b \in M$ such that:

$(\nabla)$ For all $\Delta_{0}$-formulae $\delta(x), \mathcal{M} \models \exists x \delta(x) \rightarrow \exists x<b \delta(x)$.

Note that $(\nabla)$ is equivalent to:

( $)$ For all $f \in \mathcal{F}, \mathcal{M} \models[f(0) \downarrow] \rightarrow[f(0) \downarrow]^{<b}$.

It is clear that the proof of (3) will be complete by setting $j\left(u_{k}\right)=v_{k}$ once we have two sequences $\left(u_{r}: r<\omega\right)$ and $\left(v_{r}: r<\omega\right)$ that satisfy the following four conditions:

(I) $M=\left\{u_{r}: r<\omega\right\}$.

(II) $\left\{v_{r}: r<\omega\right\}$ is an initial segment of $\mathcal{N}$, and each $v_{r}<b$.

(III) For each positive $n<\omega$, the following two properties $P(\mathbf{u}, \mathbf{v})$ and $Q(\mathbf{u}, \mathbf{v})$ hold for $\mathbf{u}=\left\langle u_{r}: r<n\right\rangle$, and $\mathbf{v}=\left\langle v_{r}: r<n\right\rangle$ :

$$
P(\mathbf{u}, \mathbf{v}) \text { : For every } f \in \mathcal{F}, \mathcal{M} \models[f(\mathbf{u}) \downarrow] \rightarrow[f(\mathbf{v}) \downarrow]^{<b} \text {. }
$$

$Q(\mathbf{u}, \mathbf{v})$ : For every $f \in \mathcal{F}$, if $\mathcal{M} \models[f(\mathbf{u}) \downarrow]$ and $f(\mathbf{u}) \notin K^{1}(\mathcal{M})$, then

$$
\mathcal{M} \models[f(\mathbf{v}) \downarrow]^{<b} \wedge f(\mathbf{u}) \neq f(\mathbf{v}) .
$$

Note that $P(\mathbf{u}, \mathbf{v})$ is equivalent to asserting that $(\exists x \delta(x, \mathbf{u}) \rightarrow \exists x<b \delta(x, \mathbf{v}))$ holds in $\mathcal{M}$ for all $\Delta_{0}$-formulae $\delta(x, \mathbf{y})$.

- Lemma 6.1.5 below enables us to carry out a routine back-and-forth construction to build sequences $\left(u_{k}: k<\omega\right)$ and $\left(v_{k}: k<\omega\right)$ that satisfy (I), (II), and (III), thereby establishing $(2) \Rightarrow(3)$ of Theorem 6.1. However, the proof of Lemma 6.1.5 is labyrinthine, so we beg for the reader's indulgence.

6.1.5. Lemma. Suppose $\mathbf{u}=\left\langle u_{r}: r<n\right\rangle$ and $\mathbf{v}=\left\langle v_{r}: r<n\right\rangle$ are in $\mathcal{M}$ with $\max (\mathbf{v})<b$, and both $P(\mathbf{u}, \mathbf{v})$ and $Q(\mathbf{u}, \mathbf{v})$ hold. Then:

(a) For every $u^{\prime} \in M$ there is $v^{\prime}<b$ such that both $P\left(\left\langle\mathbf{u}, u^{\prime}\right\rangle,\left\langle\mathbf{v}, v^{\prime}\right\rangle\right)$ and $Q\left(\left\langle\mathbf{u}, u^{\prime}\right\rangle,\left\langle\mathbf{v}, v^{\prime}\right\rangle\right)$ hold; and

(b) For every $v^{\prime} \in M$ with $v^{\prime}<\max (\mathbf{v})$ there is some $u^{\prime} \in M$ such that both $P\left(\left\langle\mathbf{u}, u^{\prime}\right\rangle,\left\langle\mathbf{v}, v^{\prime}\right\rangle\right)$ and $Q\left(\left\langle\mathbf{u}, u^{\prime}\right\rangle,\left\langle\mathbf{v}, v^{\prime}\right\rangle\right)$ hold.

Proof of (a) of Lemma 6.1.5. We begin by noting that it is well-known [26. Lem. 2] that if $P(\mathbf{u}, \mathbf{v})$ holds, then the proof of the basic Friedman 
embedding theorem as in [14, Thm. 12.3] works for countable nonstandard models of $\mathrm{I} \Sigma_{1}$ and therefore:

(1) There is a proper initial self-embedding $j_{0}$ of $\mathcal{M}$ such that $j_{0}(\mathcal{M})<b$ and $j_{0}(\mathbf{u})=\mathbf{v}$.

Given $u^{\prime} \in M$ consider the type $p(x)=p_{1}(x) \cup p_{2}(x)$, where:

$$
p_{1}(x):=\{x<b\} \cup\left\{[f(\mathbf{v}, x) \downarrow]^{<b}: f \in \mathcal{F} \text { and } \mathcal{M} \models\left[f\left(\mathbf{u}, u^{\prime}\right) \downarrow\right]\right\},
$$

and

$$
p_{2}(x):=\left\{\begin{array}{c}
{[f(\mathbf{v}, x) \downarrow]^{<b} \wedge f(\mathbf{v}, x) \neq f\left(\mathbf{u}, u^{\prime}\right):} \\
f \in \mathcal{F}, \mathcal{M} \models\left[f\left(\mathbf{u}, u^{\prime}\right) \downarrow\right] \text { and } f\left(\mathbf{u}, u^{\prime}\right) \notin K^{1}(\mathcal{M})
\end{array}\right\} .
$$

Clearly if some $v^{\prime}$ realizes $p(x)$ in $\mathcal{M}$, then $v^{\prime}<b$ and both $P\left(\left\langle\mathbf{u}, u^{\prime}\right\rangle,\left\langle\mathbf{v}, v^{\prime}\right\rangle\right)$ and $Q\left(\left\langle\mathbf{u}, u^{\prime}\right\rangle,\left\langle\mathbf{v}, v^{\prime}\right\rangle\right)$ hold. The fact that $\operatorname{Sat}_{\Sigma_{1}}$ is $\Sigma_{1}$-definable in $\mathcal{M}$, coupled with part (b) of Theorem 2.3, makes it clear that $p_{1}(x) \in \operatorname{SSy}(\mathcal{M})$. To show that $p_{2}(x) \in \operatorname{SSy}(\mathcal{M})$, let $\left\langle\delta_{i}: i \in M\right\rangle$ be a canonical enumeration of $\Delta_{0}$-formulae within $\mathcal{M}$, and let $f_{i}$ be the partial recursive function defined in $\mathcal{M}$ via:

$$
f_{i}(x)=y \text { iff } \operatorname{Sat}_{\Sigma_{1}}\left[\exists z\left(z=\mu t \delta_{i}\left(x,(t)_{0},(t)_{1}\right)\right) \wedge y=(z)_{0}\right] .
$$

Consider the subset $R$ of $\mathbb{N}$ defined as follows:

$$
R:=\left\{\langle i, j\rangle \in \mathbb{N}: \mathcal{M} \models\left[f_{j}\left(\mathbf{u}, u^{\prime}\right) \downarrow\right],\left[f_{i}(0) \downarrow\right] \text {, and } f_{j}\left(\mathbf{u}, u^{\prime}\right)=f_{i}(0)\right\} .
$$

Using the fact that $\operatorname{Sat}_{\Sigma_{1}}$ has a $\Sigma_{1}$-description one can readily verify that $R$ is the intersection with $\mathbb{N}$ of a subset of $M$ that is parametrically $\Sigma_{1}$-definable in $\mathcal{M}$, so $R \in \operatorname{SSy}(\mathcal{M})$. Moreover, using Lemma 6.1.4 we have:

$$
\begin{gathered}
\overbrace{\{j \in \mathbb{N}: \exists i\langle i, j\rangle \in R\}}^{A}= \\
\overbrace{\left\{j \in \mathbb{N}: \mathcal{M} \models\left[f_{j}\left(\mathbf{u}, u^{\prime}\right) \downarrow\right] \text { and } f_{j}\left(\mathbf{u}, u^{\prime}\right) \in K^{1}(\mathcal{M})\right\}}^{B} .
\end{gathered}
$$

Clearly $A$ is arithmetical in $R$, so $A \in \mathrm{SSy}(\mathcal{M})$ since we are assuming that $\mathbb{N}$ is strong in $\mathcal{M}$ (recall that by Theorem $2.5, \operatorname{SSy}(\mathcal{M})$ is arithmetically closed). Hence $B \in \operatorname{SSy}(\mathcal{M})$. Coupled with the closure of $\operatorname{SSy}(\mathcal{M})$ under Turing reducibility and Boolean operations, this shows that $p_{2}(x) \in \operatorname{SSy}(\mathcal{M})$, which finally makes it clear that $p(x) \in \operatorname{SSy}(\mathcal{M})$. 
On the other hand, each formula in $p_{1}(x)$ is a $\Delta_{0}$-formula (with parameters $v$ and $b$ ), and each formula in $p_{2}(x)$ is a $\Sigma_{1}$-formula (with parameters $\mathbf{u}, \mathbf{v}$, and $\left.v^{\prime}\right)$. In light of Remark 2.3.1, to show that $p(x)$ is realizable in $\mathcal{M}$ it is sufficient to verify that $p(x)$ is finitely realizable in $\mathcal{M}$.

Suppose $p(x)$ is not finitely realizable in $\mathcal{M}$. Note that the formulae in $p_{1}(x)$ are closed under conjunctions, and that by (1) $p_{1}(x)$ is finitely realizable in $\mathcal{M}$. So for some $f \in \mathcal{F}$, and some nonempty finite $\left\{g_{i}: i \leq k\right\} \subseteq$ $\mathcal{F}$ we have:

(2) $\mathcal{M} \models\left[f\left(\mathbf{u}, u^{\prime}\right) \downarrow\right]$.

(3) $\mathcal{M} \models\left[g_{i}\left(\mathbf{u}, u^{\prime}\right) \downarrow\right]$ and $g_{i}\left(\mathbf{u}, u^{\prime}\right) \notin K^{1}(\mathcal{M})$ for $i \leq k$ 凹

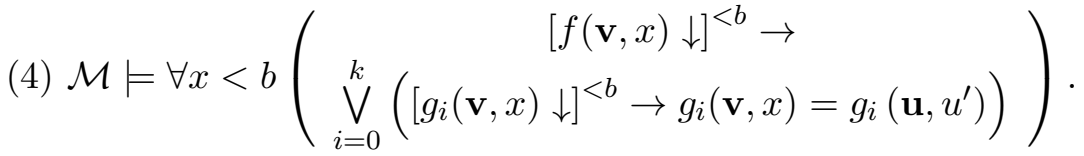

We may assume that $k$ is minimal in the sense that for any $f^{\prime} \in \mathcal{F}$ such that (2) holds with $f$ replaced by $f^{\prime}$ and any $k^{\prime}<k$, there is no subset $\left\{g_{i}^{\prime}: i \leq k^{\prime}\right\}$ of $\mathcal{F}$ which has the property that both (3) and (4) hold when $k$ is replaced by $k^{\prime}, f$ is replaced by $f^{\prime}$, and $g_{i}$ is replaced by $g_{i}^{\prime}$.

By existentially quantifying $g_{0}\left(\mathbf{u}, u^{\prime}\right), \cdots, g_{k}\left(\mathbf{u}, u^{\prime}\right)$ in (4) we obtain:

(5) $\mathcal{M} \models \exists y \theta(b, \mathbf{v}, y)$, where:

$$
\theta(b, \mathbf{v}, y):=\forall x<b\left(\begin{array}{c}
{[f(\mathbf{v}, x) \downarrow]^{<b} \rightarrow} \\
\bigvee_{i=0}^{k}\left(\left[g_{i}(\mathbf{v}, x) \downarrow\right]^{<b} \rightarrow g_{i}(\mathbf{v}, x)=(y)_{i}\right)
\end{array}\right) .
$$

At this point we wish to define functions $h_{i} \in \mathcal{F}$ for $i \leq k$. We will denote the input of each $h_{i}$ by the symbol $\diamond$ for better readability. For $i \leq k$, first let:

$$
\begin{gathered}
w_{0}(\diamond):=\mu w \exists y<w \theta(w, \diamond, y), \text { and } \\
h(\diamond):=\mu y<w_{0}(\diamond) \theta\left(w_{0}(\diamond), \diamond, y\right),
\end{gathered}
$$

and then define:

$$
h_{i}(\diamond):=(h(\diamond))_{i}
$$

\footnotetext{
${ }^{4}$ As a warm-up, the reader may first wish to focus on the special but instructive case $k=0$ in the argument that follows.
} 
Clearly for each $i \leq k, h_{i} \in \mathcal{F}$; and $w_{0}$ is well-defined iff $\left[h_{i}(\diamond) \downarrow\right]$ for each $i \leq k$. The definition of $h_{i}$ together with (5) and the assumption that $\max (\mathbf{v})<b$ makes it clear that:

(6) $\mathcal{M} \models \varphi(b, \mathbf{v})$, where $\varphi(b, \mathbf{v})$ is the formula expressing 5 :

$\forall x<b\left(\bigvee_{i=0}^{k}\left(\left(\left[g_{i}(\mathbf{v}, x) \downarrow\right]^{<b} \wedge\left[h_{i}(\mathbf{v}) \downarrow\right]^{<\max (\mathbf{v})+1}\right) \rightarrow g_{i}(\mathbf{v}, x)=h_{i}(\mathbf{v})\right)\right)$.

A salient feature of $\varphi(b, \mathbf{v})$ is that it is expressible as a $\Pi_{1}^{<b}$-formula, i.e., a formula of the form $\forall z<b \delta(\mathbf{v}, z)$, where $\delta$ is $\Delta_{0}$. Recall that by assumption $P(\mathbf{u}, \mathbf{v})$ holds, and that by contraposition $P(\mathbf{u}, \mathbf{v})$ is equivalent to:

"For all $\Delta_{0}$-formulae $\delta, \mathcal{M} \models \forall z<b \delta(\mathbf{v}, z) \rightarrow \forall z \delta(\mathbf{u}, z)$ ".

So by (6) and $P(\mathbf{u}, \mathbf{v})$, we may deduce:

$$
\mathcal{M} \models \forall x\left(\bigvee_{i=0}^{k}\left(\left(\left[g_{i}(\mathbf{u}, x) \downarrow\right] \wedge\left[h_{i}(\mathbf{u}) \downarrow\right]^{<\max (\mathbf{u})+1}\right) \rightarrow g_{i}(\mathbf{u}, x)=h_{i}(\mathbf{u})\right)\right),
$$

Recall that by $(2) \mathcal{M} \models\left[f\left(\mathbf{u}, u^{\prime}\right) \downarrow\right]$, so in light of (7) we have:

(8) $\mathcal{M} \models \bigvee_{i=0}^{k}\left(\left(\left[g_{i}\left(\mathbf{u}, u^{\prime}\right) \downarrow\right] \wedge\left[h_{i}(\mathbf{u}) \downarrow\right]^{<\max (\mathbf{u})+1}\right) \rightarrow g_{i}\left(\mathbf{u}, u^{\prime}\right)=h_{i}(\mathbf{u})\right)$.

Based on (8) we may assume without loss of generality:

(9) $\mathcal{M} \models\left(\left[g_{0}\left(\mathbf{u}, u^{\prime}\right) \downarrow\right] \wedge\left[h_{0}(\mathbf{u}) \downarrow\right]^{<\max (\mathbf{u})+1}\right) \rightarrow g_{0}\left(\mathbf{u}, u^{\prime}\right)=h_{0}(\mathbf{u})$.

At this point we claim that the following statement $(*)$ is true. Note that since the subformula marked as $\psi$ in $(*)$ (the premise of the implication) is equivalent to a formula in $p_{1}(x)$ and the index $i$ in the disjunction in $(*)$ starts from $i=1$, the veracity of $(*)$ contradicts the minimality of $k$.

$(*) \mathcal{M} \models \forall x<b\left(\begin{array}{c}\overbrace{\left(\begin{array}{c}{[f(\mathbf{v}, x) \downarrow]^{<b} \wedge\left[g_{0}(\mathbf{v}, x) \downarrow\right]^{<b} \wedge} \\ {\left[h_{0}(\mathbf{v}) \downarrow\right]^{<\max (\mathbf{v})+1} \wedge g_{0}(\mathbf{v}, x)=h_{0}(\mathbf{v})}\end{array}\right)}^{\psi} \\ \left(\bigvee_{i=1}^{k}\left[g_{i}(\mathbf{v}, x) \downarrow\right]^{<b} \rightarrow g_{i}(\mathbf{v}, x)=g_{i}\left(\mathbf{u}, u^{\prime}\right)\right)\end{array}\right)$.

Suppose to the contrary that $(*)$ fails. Then for some $c \in M$ :

\footnotetext{
${ }^{5}$ Note that a stronger form of statement (6) in which $\left[h_{i}(\mathbf{v}) \downarrow\right]^{<\max (\mathbf{v})+1}$ is weakened to $\left[h_{i}(\mathbf{v}) \downarrow\right]^{<b}$ also holds, but (6) turns out to be the appropriate ingredient for the argument that follows.
} 


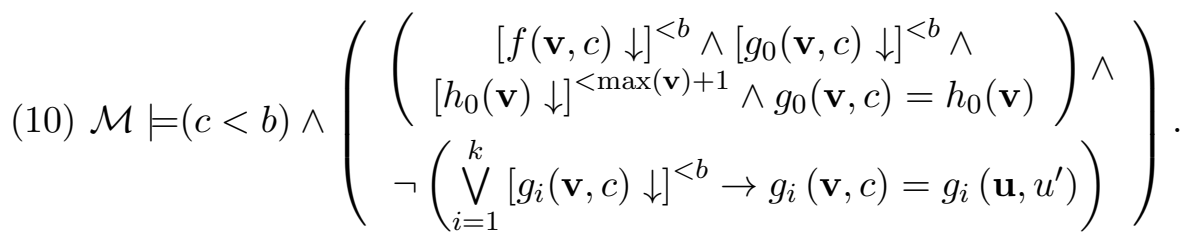

Recall that by (3) $\left[g_{0}\left(\mathbf{u}, u^{\prime}\right) \downarrow\right]$, which coupled with (10) makes it clear that:

(11) $\mathcal{M} \models\left[g_{0}\left(\mathbf{u}, u^{\prime}\right) \downarrow\right] \wedge\left[g_{0}(\mathbf{v}, c) \downarrow\right] \wedge\left[h_{0}(\mathbf{v}) \downarrow\right]^{<\max (\mathbf{v})+1}$.

In light of (11), (10), and (4) we also have:

(12) $\mathcal{M} \models h_{0}(\mathbf{v})=g_{0}(\mathbf{v}, c)=g_{0}\left(\mathbf{u}, u^{\prime}\right)$.

By $(12) \mathcal{M} \models g_{0}\left(\mathbf{u}, u^{\prime}\right)=h_{0}(\mathbf{v})$, and by $(3) g_{0}\left(\mathbf{u}, u^{\prime}\right) \notin K^{1}(\mathcal{M})$, hence:

(13) $h_{0}(\mathbf{v}) \notin K^{1}(\mathcal{M})$.

On the other hand, by $(11) \mathcal{M} \models\left[h_{0}(\mathbf{v}) \downarrow\right]^{<\max (\mathbf{v})+1}$, so (1) makes it clear that $\mathcal{M} \models\left[h_{0}(\mathbf{u}) \downarrow\right]^{<\max (\mathbf{u})+1}$. Therefore in light of (11) and (9) $g_{0}\left(\mathbf{u}, u^{\prime}\right)=h_{0}(\mathbf{u})$, so $h_{0}(\mathbf{u})=h_{0}(\mathbf{v})$, which by our assumption that $Q(\mathbf{u}, \mathbf{v})$ holds, implies $h_{0}(\mathbf{v}) \in K^{1}(\mathcal{M})$, thereby contradicting (13). This contradiction demonstrates that $(*)$ is true, thus refuting the minimality of $k$ and completing the proof.

$\square$ Lemma 6.1.5(a)

Proof of (b) of Lemma 6.1.5. The proof of this part has some resemblances to the proof of part (a), but it also exhibits certain differences. Let $\max (\mathbf{v})=v_{j}$. Then by the assumption that $P(\mathbf{u}, \mathbf{v})$ holds, $\max (\mathbf{u})=u_{j}$. Given $v^{\prime} \in M$ with $v^{\prime}<v_{j}$ consider the following type $q(x)=q_{1}(x) \cup q_{2}(x)$, where:

$$
q_{1}(x):=\left\{x<u_{j}\right\} \cup\left\{\neg[f(\mathbf{u}, x) \downarrow]: f \in \mathcal{F}, \mathcal{M} \models \neg\left[f\left(\mathbf{v}, v^{\prime}\right) \downarrow\right]^{<b}\right\},
$$

and

$$
q_{2}(x):=\left\{\begin{array}{c}
{[f(\mathbf{u}, x) \downarrow] \rightarrow f\left(\mathbf{v}, v^{\prime}\right) \neq f(\mathbf{u}, x):} \\
f \in \mathcal{F},\left[f\left(\mathbf{v}, v^{\prime}\right) \downarrow\right]^{<b}, \text { and } f\left(\mathbf{v}, v^{\prime}\right) \notin K^{1}(\mathcal{M})
\end{array}\right\} .
$$

It is routine to verify that if some element $u^{\prime}$ of $\mathcal{M}$ realizes $q(x)$, then both $P\left(\left\langle\mathbf{u}, u^{\prime}\right\rangle,\left\langle\mathbf{v}, v^{\prime}\right\rangle\right)$ and $Q\left(\left\langle\mathbf{u}, u^{\prime}\right\rangle,\left\langle\mathbf{v}, v^{\prime}\right\rangle\right)$ hold. Also one can show that $q(x) \in \operatorname{SSy}(\mathcal{M})$ using a reasoning analogous to the one used in the proof of part (a) to show that $p(x) \in \operatorname{SSy}(\mathcal{M})$. By Remark 2.3.1 to show that $q(x)$ is realized in $\mathcal{M}$ it suffices to demonstrate that $q(x)$ is finitely realizable in $\mathcal{M}$ since $q(x)$ is a short $\Pi_{1}$-type . Suppose $q(x)$ is not finitely realized in $\mathcal{M}$. Then since the formulae in $q_{1}(x)$ are closed under conjunctions and $q_{1}(x)$ is 
finitely satisfiable in $\mathcal{M}$ by statement (1) of the proof of Lemma 6.1.5(a), for some $f \in \mathcal{F}$, and some nonempty finite $\left\{g_{i}: i \leq k\right\} \subseteq \mathcal{F}$, where $k$ is minimal, we have:

(1) $\mathcal{M} \models \neg\left[f\left(\mathbf{v}, v^{\prime}\right) \downarrow\right]^{<b}$.

(2) $\mathcal{M} \models\left[g_{i}\left(\mathbf{v}, v^{\prime}\right) \downarrow\right]^{<b}$ and $g_{i}\left(\mathbf{v}, v^{\prime}\right) \notin K^{1}(\mathcal{M})$ for $i \leq k$.

(3) $\mathcal{M} \models \forall x<u_{j}\left(\begin{array}{c}{[f(\mathbf{u}, x) \downarrow] \vee} \\ \bigvee_{i=0}^{k}\left(\left[g_{i}(\mathbf{u}, x) \downarrow\right] \wedge g_{i}(\mathbf{u}, x)=g_{i}\left(\mathbf{v}, v^{\prime}\right)\right)\end{array}\right)$.

By existentially quantifying $g_{0}\left(\mathbf{v}, v^{\prime}\right), \cdots, g_{k}\left(\mathbf{v}, v^{\prime}\right)$ in (4), and taking advantage of the veracity of $\mathrm{B} \Sigma_{1}$ in $\mathcal{M}$ we obtain:

(4) $\mathcal{M} \models \exists w \exists y<w \theta(w, \mathbf{u}, y)$, where:

$$
\theta(w, \mathbf{u}, y):=\forall x<u_{j}\left(\begin{array}{c}
{[f(\mathbf{u}, x) \downarrow]^{<w} \vee} \\
\bigvee_{i=0}^{k}\left(\left[g_{i}(\mathbf{u}, x) \downarrow\right]^{<w} \wedge g_{i}(\mathbf{u}, x)=(y)_{i}\right)
\end{array}\right) .
$$

As in the proof of part(a), we will define functions $h_{i} \in \mathcal{F}$ for $i \leq k$ and will denote the input of each $h_{i}$ by the symbol $\diamond$. For $i \leq k$, first define:

$$
\begin{gathered}
w_{0}(\diamond):=\mu w \exists y<w \theta(w, \diamond, y), \text { and } \\
h(\diamond):=\mu y<w_{0}(\diamond) \theta\left(w_{0}(\diamond), \diamond, y\right),
\end{gathered}
$$

and then define:

$$
h_{i}(\diamond):=(h(\diamond))_{i}
$$

Clearly $h_{i} \in \mathcal{F}$ for $i \leq k$; and $w_{0}$ is well-defined iff $\left[h_{i}(\diamond) \downarrow\right]$ for each $i \leq k$. The definition of $h_{i}$ together with (4) yields:

(5) $\mathcal{M} \models \exists w \varphi(\mathbf{u})^{<w}$, where:

$$
\varphi(\mathbf{u}):=\forall x<u_{j}\left(\begin{array}{c}
{[f(\mathbf{u}, x) \downarrow] \vee} \\
\bigvee_{i=0}^{k}\left(\left[g_{i}(\mathbf{u}, x) \downarrow\right] \wedge\left[h_{i}(\mathbf{u}) \downarrow\right] \wedge g_{i}(\mathbf{u}, x)=h(\mathbf{u})\right)
\end{array}\right)
$$

where $\varphi(\mathbf{u})^{<w}$ is the $\Delta_{0}$-formula obtained by relativizing $\varphi(\mathbf{u})$ to the predecessors of $w$ (formally: the result of replacing every unbounded quantifier $\mathrm{Q} z$ in $\varphi(\mathbf{u})$ to $\mathrm{Q} z<w)$. Also note that $\varphi(\mathbf{u})$ can be written as a $\Sigma_{1}$-formula since $\mathcal{M} \models \mathrm{B} \Sigma_{1}$. Therefore $\mathcal{M} \models \varphi(\mathbf{v})^{<b}$ by putting (5) together with our assumption that $P(\mathbf{u}, \mathbf{v})$ holds, in other words we now have: 
(6) $\mathcal{M} \models \forall x<v_{j}\left(\bigvee_{i=0}^{k}\left(\left[g_{i}(\mathbf{v}, x) \downarrow\right]^{<b} \wedge\left[h_{i}(\mathbf{v}) \downarrow\right]^{<b} \wedge g_{i}(\mathbf{v}, x)=h_{i}(\mathbf{v})\right)\right)$.

Putting (1) together with (6) and the assumption that $v^{\prime} \leq \max (\mathbf{v})=v_{j}$ gives us:

(7) $\mathcal{M} \models \bigvee_{i=0}^{k}\left(\left[g_{i}\left(\mathbf{v}, v^{\prime}\right) \downarrow\right]^{<b} \wedge\left[h_{i}(\mathbf{v}) \downarrow\right]^{<b} \wedge g_{i}\left(\mathbf{v}, v^{\prime}\right)=h_{i}(\mathbf{v})\right)$.

Based on (7) we may assume without loss of generality:

(8) $\mathcal{M} \models\left(\left[g_{0}\left(\mathbf{v}, v^{\prime}\right) \downarrow\right] \wedge\left[h_{0}(\mathbf{v}) \downarrow\right]^{<b} \wedge g_{0}\left(\mathbf{v}, v^{\prime}\right)=h_{0}(\mathbf{v})\right)$.

At this point we claim that $(*)$ below holds. Note that $(*)$ contradicts the minimality of $k$ since the subformula marked as $\psi$ in $(*)$ (embraced by curly braces) is equivalent to the negation of a formula in $q_{1}(x)$, and in the disjunction in $(*)$ the index $i$ starts from $i=1$.

$(*) \mathcal{M} \models \forall x<u_{j}\left(\begin{array}{c}\overbrace{\left\{[f(\mathbf{u}, x) \downarrow] \vee\left(\begin{array}{c} \\ \left.\left\{g_{0}(\mathbf{u}, x) \downarrow\right] \wedge\left[h_{0}(\mathbf{u}) \downarrow\right]\right) \wedge \\ g_{0}(\mathbf{u}, x) \neq h_{0}(\mathbf{u})\end{array}\right)\right\}}^{\psi} \\ \bigvee_{i=1}^{k}\left(\left[g_{i}(\mathbf{u}, x) \downarrow\right] \wedge\left[h_{i}(\mathbf{u}) \downarrow\right] \wedge g_{i}(\mathbf{u}, x)=g_{i}\left(\mathbf{v}, v^{\prime}\right)\right)\end{array}\right)$.

Suppose to the contrary that $(*)$ fails. Then for some $c<u_{j}$ :

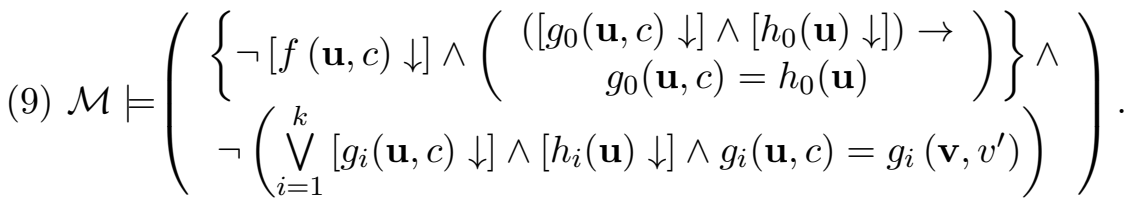

Recall that by $(2)\left[g_{0}\left(\mathbf{v}, v^{\prime}\right) \downarrow\right]$. Since $c<u_{j}$, by putting (9) together with (3) we can conclude that:

(10) $\mathcal{M} \models\left[g_{0}(\mathbf{u}, c) \downarrow\right] \wedge g_{0}(\mathbf{u}, c)=g_{0}\left(\mathbf{v}, v^{\prime}\right)$.

Also, $\mathcal{M} \models\left[h_{0}(\mathbf{u}) \downarrow\right]$ by (5) and (9). So in light of (9) and (10) we have:

(11) $\mathcal{M} \models g_{0}\left(\mathbf{v}, v^{\prime}\right)=g_{0}(\mathbf{u}, c)=h_{0}(\mathbf{u})$.

By $(11) g_{0}\left(\mathbf{v}, v^{\prime}\right)=h_{0}(\mathbf{u})$. So $h_{0}(\mathbf{u}) \notin K^{1}(\mathcal{M})$ since $g_{0}\left(\mathbf{v}, v^{\prime}\right) \notin K^{1}(\mathcal{M})$ by (2). But then we have a contradiction since by $(8) g_{0}\left(\mathbf{v}, v^{\prime}\right)=h_{0}(\mathbf{v})$, hence $h_{0}(\mathbf{v})=h_{0}(\mathbf{u})$, so $h_{0}(\mathbf{u}) \in K^{1}(\mathcal{M})$ by the assumption that $Q(\mathbf{u}, \mathbf{v})$ holds. This concludes our proof of $(*)$, which in turn contradicts the minimality of $k$ and finishes the proof.

$\square$ Lemma 6.1.5(b)

With Lemma 6.1 .5 at hand, the proof of $(2) \Rightarrow(3)$ of Theorem 6.1 is now complete. 


\section{CLOSING REMARKS AND OPEN QUESTIONS}

7.1. Remark. Let $\mathcal{L}$ be a finite extension of $\mathcal{L}_{A}$. An inspection of the proofs of Theorems 4.1, 5.1, and 6.1 make it clear that the equivalence of conditions (2) and (3) of these theorems stays valid for countable nonstandard models of $\mathrm{I} \Sigma_{1}(\mathcal{L})$. Furthermore, condition (1) of the aforementioned theorems remains equivalent to the other two conditions in the setting of $I_{1}(\mathcal{L})$ if $(1)$ is strengthened to the assertion that $j$ is a $\Delta_{0}(\mathcal{L})$-elementary self-embedding of $\mathcal{M}$.

7.2. Remark. Wilkie [25] showed that if $\mathcal{M}$ is a countable nonstandard model of PA, then:

$$
\mid\{I: I \text { is a cut of } \mathcal{M} \text { and } I \cong \mathcal{M}\} \mid=2^{\aleph_{0}} .
$$

The proof strategy in [14, Thm. $2.7(n=0)]$ of Wilkie's theorem can be shown to work for all countable nonstandard models $\mathcal{M}$ of $\mathrm{I} \Sigma_{1}$. Moreover, Theorem 4.1 can be refined by strengthening condition (3) of that theorem to state that there are $2^{\aleph_{0}}$-many cuts of $\mathcal{M}$ that can appear as the range of initial embeddings $j$ of $\mathcal{M}$ for which $I=\mathrm{I}_{\text {fix }(j)}$. These results will appear in [1].

7.3. Remark. The main results of the paper (Theorems 4.1, 5.1, and 6.1) lend themselves to a hierarchical generalization in which $\mathcal{M} \models \mathrm{I} \Sigma_{n+1}$ and the self-embedding $j$ is stipulated to be $\Sigma_{n}$-elementary. These results will also appear in [1].

7.4. Question. Is it true that in Theorems 5.1 and 6.1 condition (3) can be strengthened by adding that there are continuum-many cuts of $\mathcal{M}$ that can be realized as the range of $j$ ?

Remark 7.1 suggests that Question 7.4 has a positive answer.

7.5. Question. Is there some $n \in \omega$ such that every countable nonstandard model of $\mathrm{I} \Sigma_{n}$ has a contractive (i.e., $j(a) \leq a$ always) proper initial selfembedding? And if the answer is positive, what is the minimal such $n$ ?

The above question is motivated by Corollary 3.4.2.

7.6. Question. Suppose $\mathcal{M}$ is a countable nonstandard model of $\mathrm{I}_{1}$ in which $\mathbb{N}$ is a strong cut. Is every proper $\Sigma_{1}$-elementary submodel of $\mathcal{M}$ isomorphic to $\operatorname{Fix}(j)$ for some $j \in \operatorname{PISE}(\mathcal{M})$ ? 
The above question is prompted by the result mentioned in footnote 3 , and the fact that the proof of Theorem 6.1 makes it clear that the theorem remains valid if in conditions and (1) and (3) of the statements of that theorem, the requirement that $\operatorname{Fix}(j)=K^{1}(\mathcal{M})$ is modified to $\operatorname{Fix}(j)=$ $K^{1}(\mathcal{M}, m)$, where $m \in M$.

7.7. Question. Suppose $I$ is a strong cut of $\mathcal{M} \models I \Sigma_{1}, \mathcal{N} \prec_{\Sigma_{1}} \mathcal{M}$, and $\mathcal{N}$ is $I$-coded (i.e., there is an element $s$ of $\mathcal{M}$ such that $N=\left\{(s)_{i}: i \in I\right\}$ and $s_{i} \neq s_{j}$ if $\left.i<j \in I\right)$, then $\mathcal{N}$ can be realized as $\operatorname{Fix}(j)$ for some $j \in \operatorname{PISE}(\mathcal{M})$ ?

The impetus for the above question can be found in [7, Thm. 4.5.1].

\section{References}

[1] S. Bahrami, doctoral dissertation, Tarbiat Modares University (in preparation).

[2] P. D'Aquino and J. Knight, Coding in $\mathrm{I} \Delta_{0}$, Nonstandard Models of Arithmetic and Set theory, pp. 23-36, Contemp. Math., 361, Amer. Math. Soc., Providence, RI, 2004.

[3] C. Dimitracopoulos and J. Paris, A note on a theorem of H. Friedman, Math. Logic Quart. 34 (1988), pp. 13-17.

[4] C. Dimitracopoulos and H. Gaifman, Fragments of Peano's Arithmetic and the MRDP theorem, in Logic and Algorithmic, Monogr. Enseign. Math. University of Geneva, 1982, pp. 187-206.

[5] A. Enayat, From bounded arithmetic to second order arithmetic via automorphisms, in Logic in Tehran, Lecture Notes in Logic, 26, Association for Symbolic Logic, 2006, pp. 87-113.

[6] ________ Automorphisms of models of bounded arithmetic, Fund. Math. 192 (2006), pp. 37-65.

[7] _______ Automorphisms of models of arithmetic: a unified view, Ann. Pure Appl. Logic 145 (2007), pp. 16-36.

[8] _______ A new proof of Tanaka's theorem, in New Studies in Weak Arithmetics, CSLI Lectures Notes 11, Stanford, 2014, pp. 93102. 
[9] A. Enayat and T. L. Wong, Unifying the model theory of first-order and second-order arithmetic via $\mathrm{WKL}_{0}^{*}$, Ann. Pure Appl. Logic, 168 (2017), pp. 1247-1283.

[10] H. Friedman, Countable models of set theories, Lecture Notes in Math. 337, Springer, Berlin, 1973, pp. 539-573.

[11] H. Gaifman, Models and types of arithmetic, Ann. Math. Logic 9 (1976), pp. 223-306.

[12] P. Hájek and P. Pudlák, Two orderings of the class of all countable models of Peano arithmetic, Model theory of algebra and arithmetic, Lecture Notes in Math. 834, 1980, pp. 174-185.

[13] _____________ Metamathematics of First Order Arithmetic, Springer, Heidelberg, 1993.

[14] R. Kaye, Models of Peano Arithmetic, Oxford University Press, Oxford, 1991.

[15] R. Kaye, R. Kossak, and H. Kotlarski, Automorphisms of recursively saturated models of arithmetic, Ann. Pure Appl. Logic 55 (1991), pp. 67-99.

[16] R. Kossak and J. Schmerl, The Structure of Models of Peano Arithmetic, Oxford, 2006.

[17] Z. Mijajlović, Submodels and definable points in models of Peano arithmetic, Notre Dame J. Formal Logic 24 (1983), pp. 417-425.

[18] J. Paris and L. Kirby, $\Sigma_{n}$-collection schemas in arithmetic, in Logic Colloquium '77, North-Holland Publishing Company, Amsterdam, 1978, pp. 199-209.

[19] J.-P. Ressayre, Nonstandard universes with strong embeddings, and their finite approximations, Logic and combinatorics, Contemp. Math. 65, Amer. Math. Soc., Providence, RI, 1987, pp. 333-358.

[20] S. Simpson, Subsystems of Second Order Arithmetic, SpringerVerlag, Heidelberg, 1999.

[21] C. Smoryński, Nonstandard models of arithmetic, Course Notes, Utrecht University, Fall 1978, http://igitur-archive.library.uu.nl/ph/2010-1214-200237/preprint289.pdf 

Recursively saturated nonstandard models of arithmetic,

J. Symb. Logic 46 (1981), pp. 259-286.

[23] ________ Back-and-forth in a recursively saturated model of arithmetic, Logic Colloquium '80, North-Holland, Amsterdam, 1982, pp. 273-278.

[24] __________ Lectures on nonstandard models of arithmetic, Logic Colloquium '82, North-Holland, Amsterdam, 1984, pp. 1-70.

[25] A. Wilkie, Models of Number Theory, doctoral dissertation, University of London, 1973.

[26] K. Yokoyama, Notes on Friedman's self-embedding theorem, Studies in Weak Arithmetics 3, CSLI Lectures Notes 217, 2016, Stanford, pp. 231-241.

Saeideh Bahrami

Dept. of Mathematics

Tarbiat Modares University

P.O. Box 14115-111, Tehran, Iran

E-mail: bahrami.saeideh@gmail.com
Ali Enayat

Dept. of Philosophy, Linguistics, \& Theory of Science University of Gothenburg

Box 200, SE 405 30, Gothenburg, Sweden

E-mail: ali.enayat@gu.se 